\title{
Rapid Noninvasive Skin Monitoring by Surface Mass Recording and Data Learning
}

\author{
Yingdi Zhu, Andreas Lesch, Xiaoyun Li, Tzu-En Lin, Natalia Gasilova, Milica Jović, Horst Matthias Pick,
} Ping-Chih Ho, and Hubert H. Girault*

Cite This: JACS Au 2021, 1, 598-611

Read Online

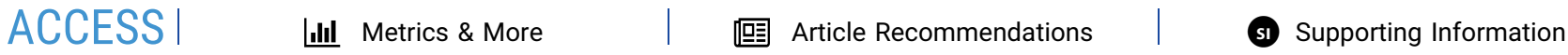

ABSTRACT: Skin problems are often overlooked due to a lack of robust and patient-friendly monitoring tools. Herein, we report a rapid, noninvasive, and high-throughput analytical chemical methodology, aiming at real-time monitoring of skin conditions and early detection of skin disorders. Within this methodology, adhesive sampling and laser desorption ionization mass spectrometry are coordinated to record skin surface molecular mass in minutes. Automated result interpretation is achieved by data learning, using similarity scoring and machine learning algorithms. Feasibility of the methodology has been demonstrated after testing a total of 117 healthy, benign-disordered, or malignant-disordered skins. Remarkably, skin malignancy, using melanoma as a proof of concept, was

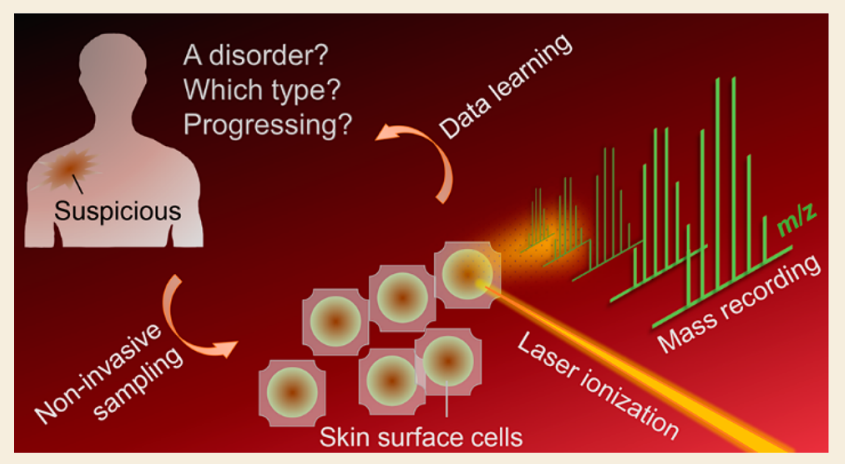
detected with $100 \%$ accuracy already at early stages when the lesions were submillimeter-sized, far beyond the detection limit of most existing noninvasive diagnosis tools. Moreover, the malignancy development over time has also been monitored successfully, showing the potential to predict skin disorder progression. Capable of detecting skin alterations at the molecular level in a nonsurgical and time-saving manner, this analytical chemistry platform is promising to build personalized skin care.

KEYWORDS: skin disorder, noninvasive diagnosis, mass spectrometry, similarity scoring, machine learning

\section{INTRODUCTION}

Human skin acts as a barrier to protect the body from the environment. As the largest organ of the body, the skin is at especially high risk of suffering from harmful impacts both externally and internally. Skin disorders are thus some of the most common human health problems worldwide. The disorder can result from diverse factors, like wounding, infection, allergy, inflammation, genetic mutation, and so forth. ${ }^{1}$ Some inner body health problems can also cause symptoms on the skin. For instance, immune system abnormalities, cardiovascular diseases, neurological diseases, diabetes, and microbial infections like the current COVID-19 epidemic can trigger specific physical or chemical changes on the skin. ${ }^{2,3}$ Many skin disorders could be life-threatening if not treated properly in time. Taking skin cancer as an example, the cancer cells can spread from where they arise, normally in the epidermis or the epidermal-dermal junction, deep into the dermis and further invade other parts of the body, resulting in a dangerous situation with a high fatality rate. ${ }^{4} \mathrm{~A}$ regular monitoring of the skin status, therefore, is of great value for the public health policy strategy.

Clinically, skin monitoring mainly relies on microscopic, imaging, and molecular tools. The initial screening is often based on visual and physical evaluations, noting the skin lesion size, shape, color, texture, evolving, as well as the occurrence of bleeding, oozing or crusting, using tools like dermoscopy. Once suspected as a skin malignancy, a surgical biopsy is carried out to establish a definitive diagnosis. During the process, a small amount of skin tissue is removed from the suspicious region and analyzed using histopathological or genetic techniques like hematoxylin-eosin staining, immunohistochemistry, in situ hybridization, and gene expression profiling. ${ }^{5,6}$ Lymph node biopsy is conducted when there are signs of malignant spread. Body imaging tests are ordered to check for metastasis at distant sites, using tools like computed tomography, ultrasonography, or magnetic resonance imaging. ${ }^{7}$ Over the years, more analytical tools have been proposed to analyze biopsied skin samples. Examples include Raman spectroscopy, scanning electrochemical microscopy, quantitative chemical sensors, quantitative proteomics, and mass spectrometry imaging, to mention a few..$^{8-10}$ Despite these

Received: November 13, 2020

Published: March 22, 2021 

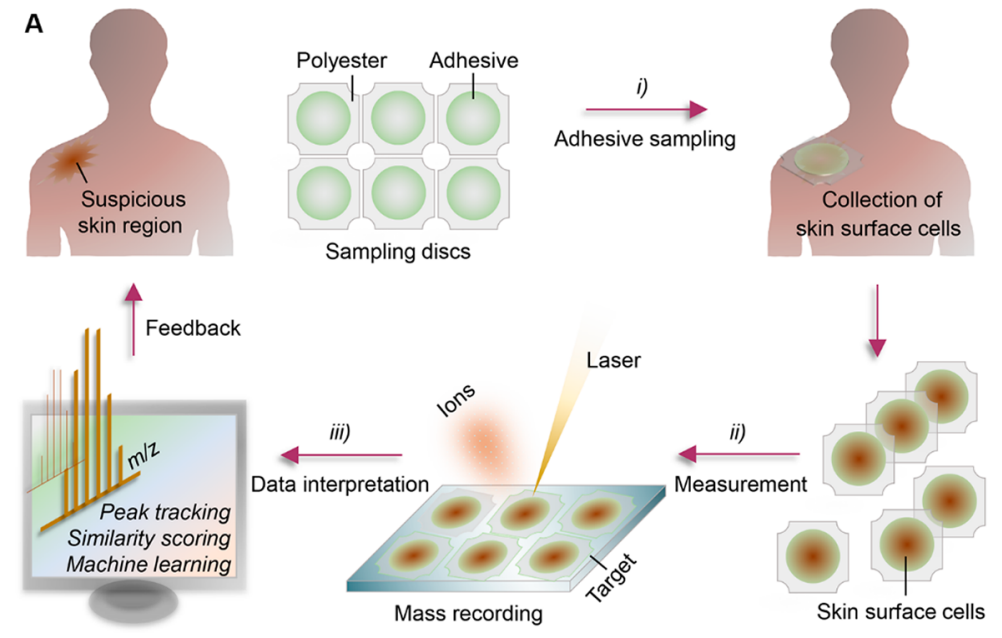

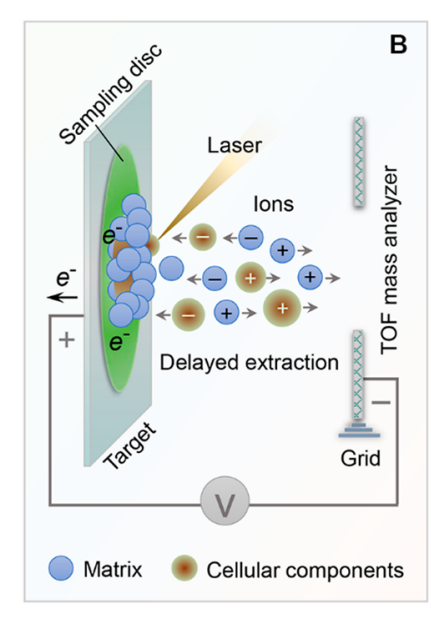

Figure 1. Methodology description. (A) Schematic illustration of the proposed methodology for skin monitoring and diagnosis. (B) Illustration of the mass spectrometry ionization process with the presence of a sampling disc. All of the measurements were conducted under linear positive mode with $400 \mathrm{~ns}$ delayed extraction, using sinapinic acid as the matrix.

advancements, strategies that are not only robust but also rapid and patient-friendly are in short supply.

Herein, we develop an analytical chemical methodology to achieve a quick, noninvasive, and high-throughput skin monitoring. Within it, a well-established adhesive sampling procedure is used in conjunction with matrix-assisted laser desorption ionization time-of-flight (MALDI-TOF) mass spectrometry to record skin surface mass profiles. The mass profiles, determined by the skin molecular composition, can act as "fingerprints" for the characterization of the skin state. They are afterward subjected to similarity scoring and machine learning to reach an automated result interpretation. The adhesive sampling provides a mild collection of cells from epidermal skin layer using adhesive discs. This sampling procedure is noninvasive, painless, readily performed, and has been widely accepted in skin pharmacological and physiological studies. ${ }^{11,12}$ MALDI-TOF mass spectrometry (MS) is now implemented in clinical laboratories for a broad range of applications such as microbial identification. It detects analytes precisely according to their molecular weights with the turnaround time of several minutes only. The mass spectrometer target is designed to accommodate hundreds of sample spots, so that the assays can be conducted in a high-throughput manner. The mass spectral data are easy to process due to the presence of mostly singly charged analyte ions. ${ }^{13,14}$ Automated data interpretation, using artificial intelligence approaches like machine learning, data mining, or complex network analysis, is one of the most prospective trends in clinical diagnosis. ${ }^{15}$ It eliminates the heavy reliance on skilled physicians with prior clinical experience and enables the processing of large complex data sets in a short time, e.g., analysis of hundreds of mass spectra in milliseconds. After testing on a total of 117 skin samples, the developed methodology is demonstrated to allow a routine examination of skin state, an early and accurate detection of skin disorders, and a dynamic monitoring of the disorder progression.

\section{RESULTS}

\section{Methodology Description}

As illustrated in Figure 1A, the methodology is composed of three steps, including (i) adhesive collection of cells from the surface of suspicious skin regions using sterile adhesive sampling discs ("sampling"), (ii) recording mass fingerprints of the collected skin cells on the sampling discs by MALDITOF mass spectrometry ("measurement"), and (iii) interpretation of the skin surface mass fingerprint data using similarity scoring algorithms, peak tracking method, and machine learning tools ("data interpretation"). The whole detection process is rather rapid, with each step taking only a few minutes.

The sampling discs used herein (photo in Figure S1) have been validated by human skin safety tests. The discs carrying the collected skin cells were directly covered with the MALDI matrix for the mass recording. This sample preparation process is straightforward, without any pretreatment like cell lysis, component extraction, or analyte labeling. During the MALDI measurements, the presence of a sampling disc made of nonconducting materials would hinder the electrical charge dissipation from the sample surface and thus affect the ionization process (Figure 1B). ${ }^{16}$ This could result in a decrease of absolute peak intensities, as observed during a comparative test with in vitro grown cells (Figure S2). Nevertheless, the mass spectra still displayed a good mass resolution with a high signal-to-noise ratio. For each investigated skin region, the mass spectrum was obtained with $5 \times 250$ laser shots throughout the sample region to reduce the possible impact from "sweet" spots and to generate a panorama of the whole skin region. For intersample comparison, the normalized spectra with relative peak intensities were considered to minimize the influence from sample quality or experimental conditions.

\section{Tests on Healthy Human Skin and Benign Skin Disorders}

The methodology was first tested on a normal skin region, i.e., a region without moles, scars, pimples, hair, etc., on the forearm of a healthy adult volunteer. The skin region was sampled four times in a row, using a new disc each time. Stratified cells with a lateral diameter of $\sim 30 \mu \mathrm{m}$ were clearly observed on each disc (Figure 2A-D). The collected cell layer had a mean thickness of $\sim 3 \mu \mathrm{m}$ (height profile in Figure 2E). Most of the cells were dead, as confirmed by trypan blue staining of a freshly collected sample (Figure 2F). The cells should be derived from stratum corneum, the skin layer composed of 

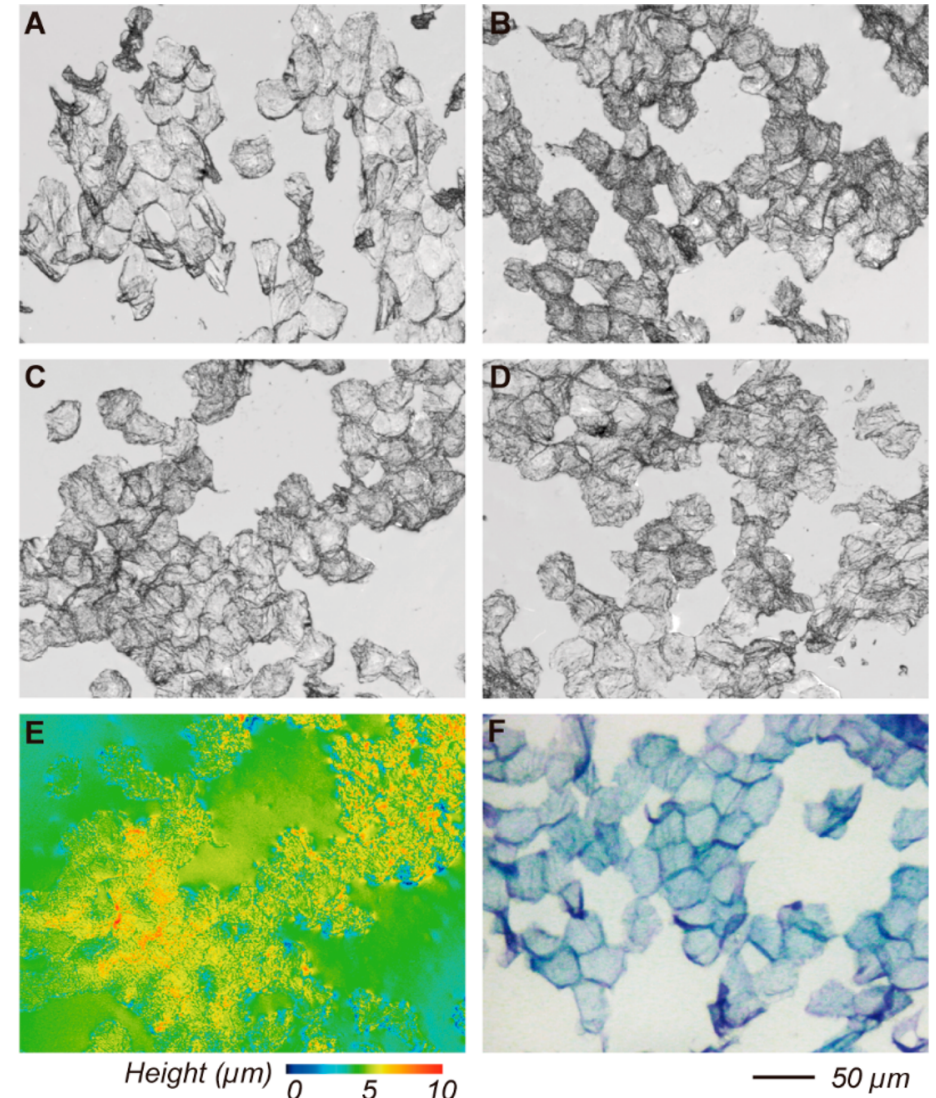

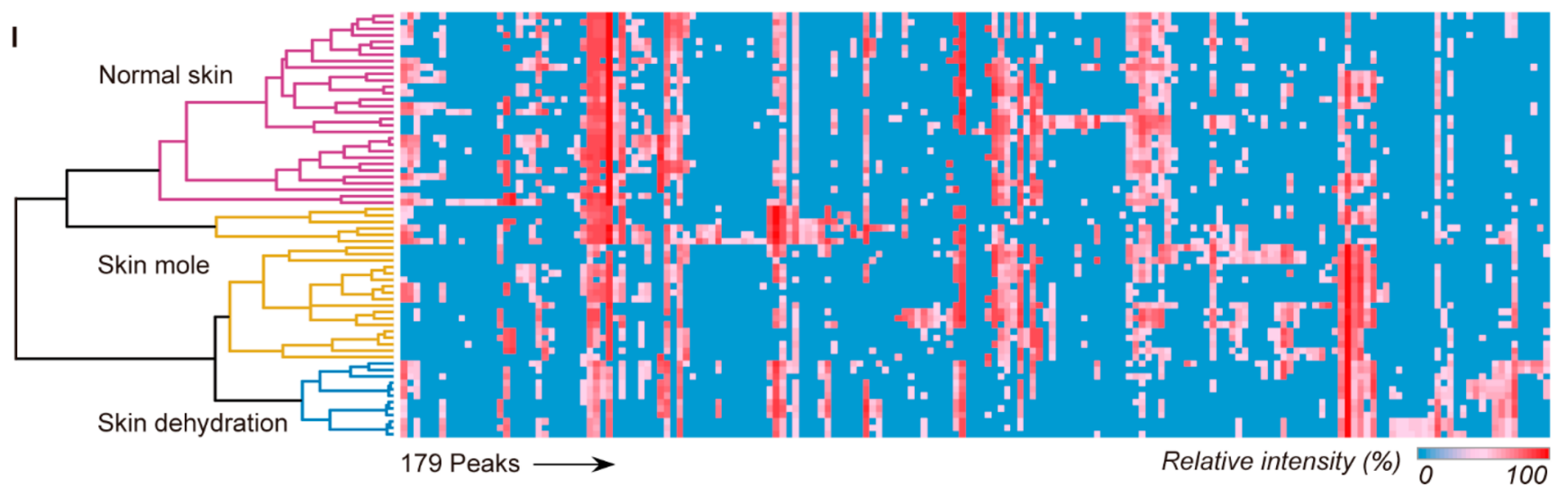

Figure 2. Tests on healthy human skin and benign human skin disorders. (A-J) Characterization of a normal skin region on the forearm of a healthy adult volunteer with four times adhesively sampling in a row: (A-D) 3D laser scanning microscopic images from the four samples, (E) microscopic height profile of the third sample, (F) microscopic image after trypan blue staining (10 min immersion in $0.02 \%$ trypan blue) of a freshly collected sample, and $(\mathrm{G})$ mass fingerprints from the four samples. (H,I) Analysis of skin surface mass fingerprints from three skin conditions, i.e., normal healthy skins, skin moles, and dry-itchy dehydrated skin, derived from different healthy volunteers at different body sites: $\mathrm{(H)}$ averaged mass fingerprint at each skin condition (shaded area: interquartile range) and (I) hierarchical clustering using cosine similarity and average linkage, correlated with the fingerprint peak intensity profiles.

dead corneocytes with a thickness between 10 and $40 \mu \mathrm{m}$ on healthy humans. ${ }^{17}$ Hence, the sampling process is noninvasive and painless, as the removal of corneocytes will not damage the deep skin tissue, and the removed cells will be regenerated naturally within around 14 days. ${ }^{18}$ Mass fingerprints generated from the four sequential samplings closely resembled each other, displaying mutual cosine similarity of $0.912( \pm 0.037)$ (Figure 2G). The cosine similarity was calculated using the algorithm of cosine correlation. It considers both the location (mass-to-charge ratio, $\mathrm{m} / \mathrm{z}$ ) and the relative intensity of each mass spectral peak, scoring spectral similarity between 0 (completely different) and 1 (totally identical). The result indicates that a suspicious skin region can be sampled multiple times to ensure reliability of the detection. The detectable mass peaks were mainly located in the mass range of 2000-20,000 $m / z$, mostly derived from proteins in the skin cells. Notably, the sampling disc itself did not bring any interference mass signal (Figure S3).

The methodology is able to distinguish between normal healthy skin and benign skin disorders, as demonstrated by testing 66 skin regions from nine healthy adult volunteers. The volunteers, aged between 25 and 63, came from different 
countries in Asia, Europe, and South America. The test was conducted on three common skin conditions, i.e., normal healthy skin, skin moles, and dehydrated skin, at different body sites, i.e., hand, arm, neck, chest, waist, and leg (Table S1). A skin mole occurs when melanocytes, the pigment-producing skin cells, grow in a cluster instead of spreading throughout the epidermis. All the tested moles have been confirmed as benign by a local dermatologist. The investigated dehydrated skins were characterized by the symptoms of dryness, itching, and slightly cracking. The dehydration was mostly caused by the cold winter weather with low humidity, typical in Alpine Switzerland where the volunteers currently live. Mass fingerprints generated from all the investigated skins are shown in Figure S4, with the averaged spectrum (with interquartile range) at each condition displayed in Figure $2 \mathrm{H}$. In order to explore their intercorrelations, hierarchical cluster analysis (HCA) was conducted using the spectral cosine similarity. HCA is an unsupervised machine learning method to group objects in such a way that the objects in the same group are more similar to each other than to those in other groups. Results showed that mass fingerprints derived from the same skin condition were generally more similar to each other than to fingerprints from other conditions, with corresponding characteristic peak distribution profiles (Figure 2I, with peak details in Table S2). Specifically, mass fingerprints from normal healthy skin displayed mutual spectral cosine similarity of $0.861( \pm 0.058)$, with a 60 POP common peak rate (the ratio between the number of peaks detected with more than $60 \%$ of presence among the mass spectra and the averaged peak number per spectrum) reaching $76 \%$ (34/45). This high similarity is consistent with the fact that molecular compositions are generally conserved among healthy humans. ${ }^{19}$ The slight variations among samples could come from the differences in age, gender, skin tone, or body site (investigation in Figure S5). The occurrence of skin dehydration or skin moles changed the epidermal constituents and thus made the mass fingerprints different, with the similarity to normal healthy skins decreased to $0.299( \pm 0.101)$ and $0.519( \pm 0.143)$, respectively. The dehydrated skin regions were generally similar to each other in morphology and shared mutual similarity as high as $0.890( \pm 0.054)$, with a $60 \mathrm{POP}$ common peak rate of $76 \%(35 / 46)$. The mutual similarity among the skin moles was lower, i.e., $0.664( \pm 0.216)$, with a 60 POP common peak rate of $66 \%(31 / 47)$. Some moles even displayed higher similarity to their corresponding normal skins than to the moles from other individuals. This could be explained by their difference in morphology, with the lateral diameter ranging from 1 to $5 \mathrm{~mm}$ and the color increasing from light brown to dark black.

\section{Detection and Monitoring of Malignant Skin Disorder in} Mice

Feasibility of the methodology was further demonstrated through a systematic in vivo study on a malignant skin disorder, i.e., melanoma-type skin cancer. Because of its propensity for lethal metastasis and therapeutic resistance, melanoma is one of the deadliest forms of human malignancy. ${ }^{20}$ Due to ethical considerations, the study was conducted on mouse models, instead of on human patients directly. The malignancy was induced using a well-developed genetically engineered method, by treating Tyr::CreER;BRaf $^{\text {CA }}$;Pten ${ }^{\text {lox } 4-5}$ transgenic mice with 4-hydroxytamoxifen (4-HT) topically on the back skin. ${ }^{21,22}$ This cancer model carried the most common genetic mutation in human melanoma and had the potential of metastatic outgrowth. By controlling the tumor growth time, the mice developed skin cancer at different progression stages, including (i) pretumor lesions with submillimeter-sized black speckles, (ii) radial growth phase tumors with cancer cells confined in the epidermis and epidermal-dermal junction, (iii) vertical growth phase tumors with cancer cells invading the dermis, and (iv) metastatic tumors with cancer cells invading lymph nodes and

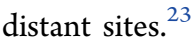

Adhesive sampling was applied to the 4-HT-treated skin regions after optimization of the sampling procedure (Figures S6 and S7). Representative mass fingerprints generated throughout the disorder progression are shown in Figure 3. Among them, Figure 3A, more in Figure S8, was from healthy controls. Figure 3B,C was from the fourth week after 4-HT administration, when the cancer was successfully initiated in the skin with the development of submillimeter-sized black speckles. The speckle number varied among mice, implying the individual difference in the cancer initiation dynamics. Figure $3 \mathrm{D}-\mathrm{F}$ was from the sixth week, when small tumor nodules formed, with the superficial surface area between 10 and 24 $\mathrm{mm}^{2}$ among mouse individuals. Figure $3 \mathrm{G}, \mathrm{H}$ came from the seventh week, when the tumor size increased to $50-70 \mathrm{~mm}^{2}$. Figure 3I-L came from the ninth week, when the tumors reached $130-182 \mathrm{~mm}^{2}$ and started metastasis, as indicated by the emergence of new tumor nodules located more than 15 $\mathrm{mm}$ away from the primary tumors. ${ }^{24}$ Along with the disorder progression, the fingerprint patterns changed gradually, with difference observed on peaks like Peak $1(4690 \mathrm{~m} / z)$, Peak 2 $(7493 \mathrm{~m} / z)$, Peak $3(7811 \mathrm{~m} / z)$, Peak $4(8712 \mathrm{~m} / z)$, Peak 5 $(9366 \mathrm{~m} / z)$, Peak $6(9979 \mathrm{~m} / z)$, Peak $7(10,165 \mathrm{~m} / z)$, Peak 8 $(10,598 \mathrm{~m} / z)$, Peak $9(10,871 \mathrm{~m} / z)$, Peak $10(11,546 \mathrm{~m} / z)$, Peak $11(14,975 \mathrm{~m} / z)$, and Peak $12(15,615 \mathrm{~m} / z)( \pm 1000$ ppm mass tolerance). These peaks will be discussed with more details later. Due to the accumulation of melanin pigment produced by the cancer cells, the lesions were typically blueblack in color (representative photos in Figure S9). Compared to the healthy controls, the collected skin cells displayed obvious difference in size and shape when the tumor size was larger than $70 \mathrm{~mm}^{2}$ (microscopic images in Figure S10). The sampling from the tumor lesions was estimated to collect cancer cells together with the surrounding texture, like the resident and infiltrating host cells, secreted factors, and extracellular matrix proteins.

An automated result interpretation, using data learning tools, showed that the disorder could be detected at early stages with high confidence. Figure 4A displays the correlations among the skin surface mass fingerprints during the disorder progression, obtained from hierarchical clustering based on fingerprint cosine similarity (cluster distance in Figure S11, similarity scores in Table S3). The similarity among all healthy skins was higher than 0.960 , with a mean value of $0.981( \pm 0.007)$. When the cancer happened, the mass fingerprints were found to be different from the healthy cluster with the similarity decreased, more specifically $0.865( \pm 0.013)$ for the very early lesions with submillimeter-sized speckles and $0.153( \pm 0.059)$ for the $10-$ $182 \mathrm{~mm}^{2}$ tumors. At early stages with speckles only, although the similarity to healthy skin was not as low as that from tumors, the fingerprint peaks were considerably different from the healthy ones with $35 \%$ (32/91 peaks) newly detected or increased in intensity and $9 \%$ (8/91 peaks) disappeared or decreased in intensity (absolute log 2 -fold change $\geq 1$, paired $t$ 
A

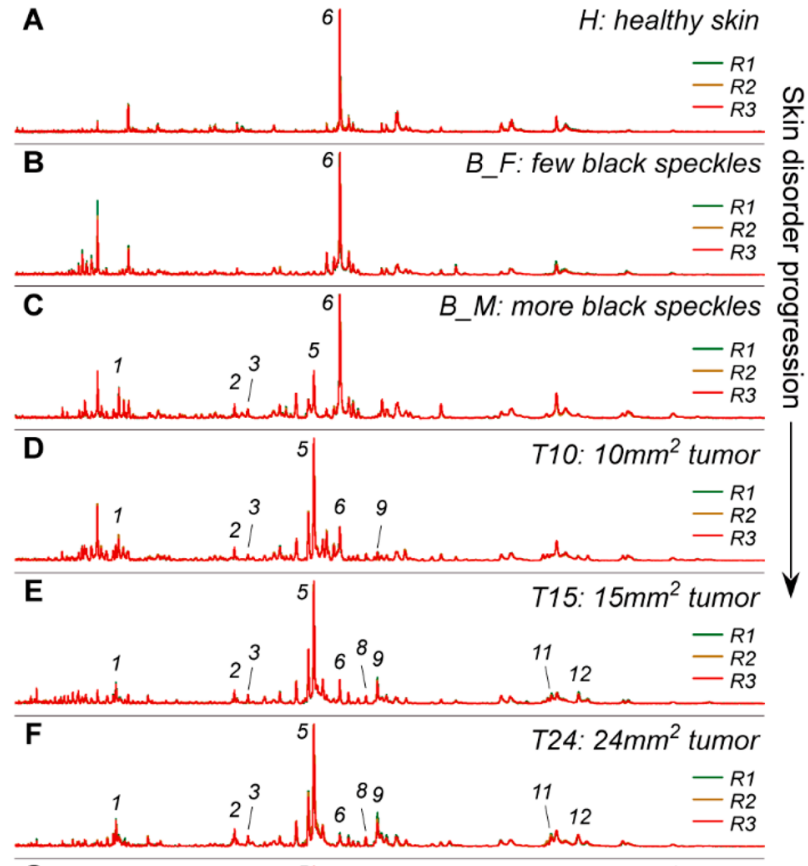

G
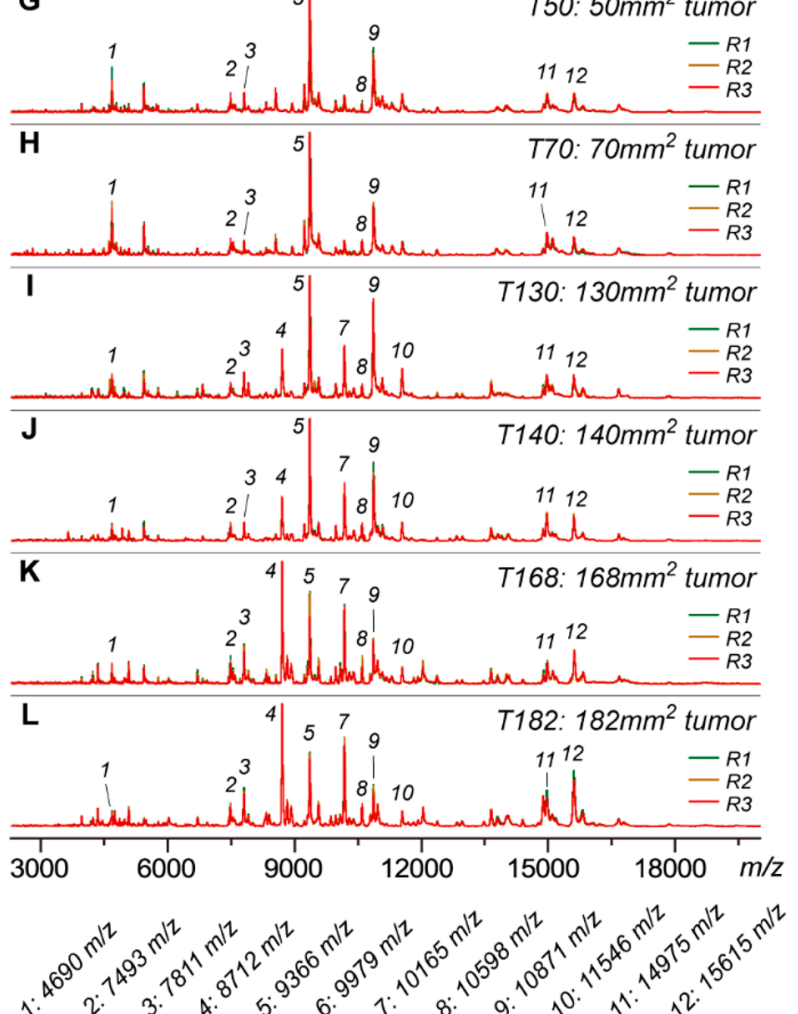

Figure 3. Representative mass fingerprints during malignant skin disordering. The skin conditions are $(A)$ healthy, $(B, C)$ lesions with different number of black speckles $\left(B_{-} F, B_{-} M\right)$, (D-L) tumor lesions with superficial surface area of $10-182 \mathrm{~mm}^{2}(T 10, T 15, T 24, T 50$, T70, T130, T140, T168, T182). Mass peaks associated with the malignancy growth are labeled in order of their $m / z$ values. All spectra were obtained from the fourth samplings on the skin regions treated with 4-HT.

test $P$ value $\leq 0.05$ ) (Figure 4B, peak list in Table S4). Many of these different peaks were detected in overall low intensity and thus made a limited contribution to the fingerprint cosine similarity. If compared in a way that increases the weighting of peak presence but reduces or eliminates the weighting of peak intensity, the differentiation between the speckle lesions and healthy skins is expected to be easier. ${ }^{25}$ The algorithm of relative Euclidean distance and Jaccard index were proposed as such measures, with the similarity scores decreased to $0.326( \pm 0.049)$ and $0.446( \pm 0.058)$, respectively (Figure $4 \mathrm{C})$. Both scores were significantly lower $\left(t\right.$ test $P$ value $4 \times 10^{-70}, 9$ $\times 10^{-70}$ ) than those of their counterpart among the healthy skins, i.e., $0.679( \pm 0.072)$ and $0.810( \pm 0.070)$, respectively (more investigation in Figures $\mathrm{S} 12$ and S13). To further evaluate the detection performance, the mass fingerprints were subjected to machine learning classification. Fingerprints from altogether 51 skin samples were investigated, 18 healthy and 33 cancerous (including speckle and tumor lesions at different progression stages). After a total of 84 classifiers from commonly used learning models were screened (on a Weka machine learning workbench), ${ }^{26} 28$ of them produced satisfactory results, with the detection accuracy (correct classification rate) higher than 0.98, the kappa statistic (a metric comparing observed accuracy with expected accuracy) higher than 0.96, and other performance measures like Fmeasure (the weighted harmonic mean of precision and recall) and ROC area (area under the receiver operating characteristic curve) higher than 0.98 (10-fold cross-validation) (Figure 4D, Table S5). Notably, 12 of these classifiers even provided $100 \%$ correct detection (true positive rate 1 , false positive rate 0 , for both healthy and cancerous skin) with all the performance measures reaching 1 . They were MultiClassClassifier (MCC), KStar, IB1, Winnow, SMO, Logistic, LibSVM, HNB, BayesianLogisticRegression, WAODE, AODEsr, and AODE. These classifiers could be the top choices for a diagnosis in the future. Such outcome also demonstrated the robustness of the proposed data collection procedure for skin disorder detection, as it showed no strong preference on the classification algorithms for the data interpretation. The limit of detection for the skin cancer was thus displayed as early as the occurrence of submillimeter-sized black speckles. Such a detection limit is much better than many existing noninvasive methods, for instance, the widely used "ABCDE" dermoscopic examinations often requiring cancer lesions larger than $6 \mathrm{~mm}$ in diameter $\left(\sim 30 \mathrm{~mm}^{2}\right.$ area $){ }^{27}$

More than detection of the disorder, progression of the disorder over time was also monitored with convenience by the new methodology. As shown in Figure 4A, along with the disorder development, the skin lesions with speckles, early tumors $\left(10-24 \mathrm{~mm}^{2}\right)$, medium-sized tumors $\left(50-70 \mathrm{~mm}^{2}\right)$, and further metastatic tumors (130-182 $\left.\mathrm{mm}^{2}\right)$ deviated gradually from the healthy cluster, with cosine similarity to the healthy decreased to $0.865( \pm 0.013), 0.205( \pm 0.082)$, $0.113( \pm 0.014)$, and $0.134( \pm 0.010)$, respectively. It was also found that lesions at the same or nearby progression stages had more similar mass fingerprints compared to the lesions at stages far away from each other. For instance, the $10 \mathrm{~mm}^{2}$ early skin tumor displayed fingerprint cosine similarity of $0.888( \pm 0.012)$ to other early tumors $\left(10-24 \mathrm{~mm}^{2}\right)$, while its similarity to the earlier stage (speckles), the later stage (medium-sized tumors), and further to the metastatic tumors was decreased to $0.542( \pm 0.095), 0.776( \pm 0.011)$, and $0.537( \pm 0.118)$, respectively. Such findings were further confirmed by principal component analysis (PCA), another type of unsupervised machine learning. PCA explains the variation of a large number of original responses (here, the hundreds of mass fingerprint peaks) using a smaller number of 
A Cosine similarity \begin{tabular}{llllll}
\hline & & & & \\
0.0 & 0.2 & 0.4 & 0.6 & 0.8 & 1.0
\end{tabular}

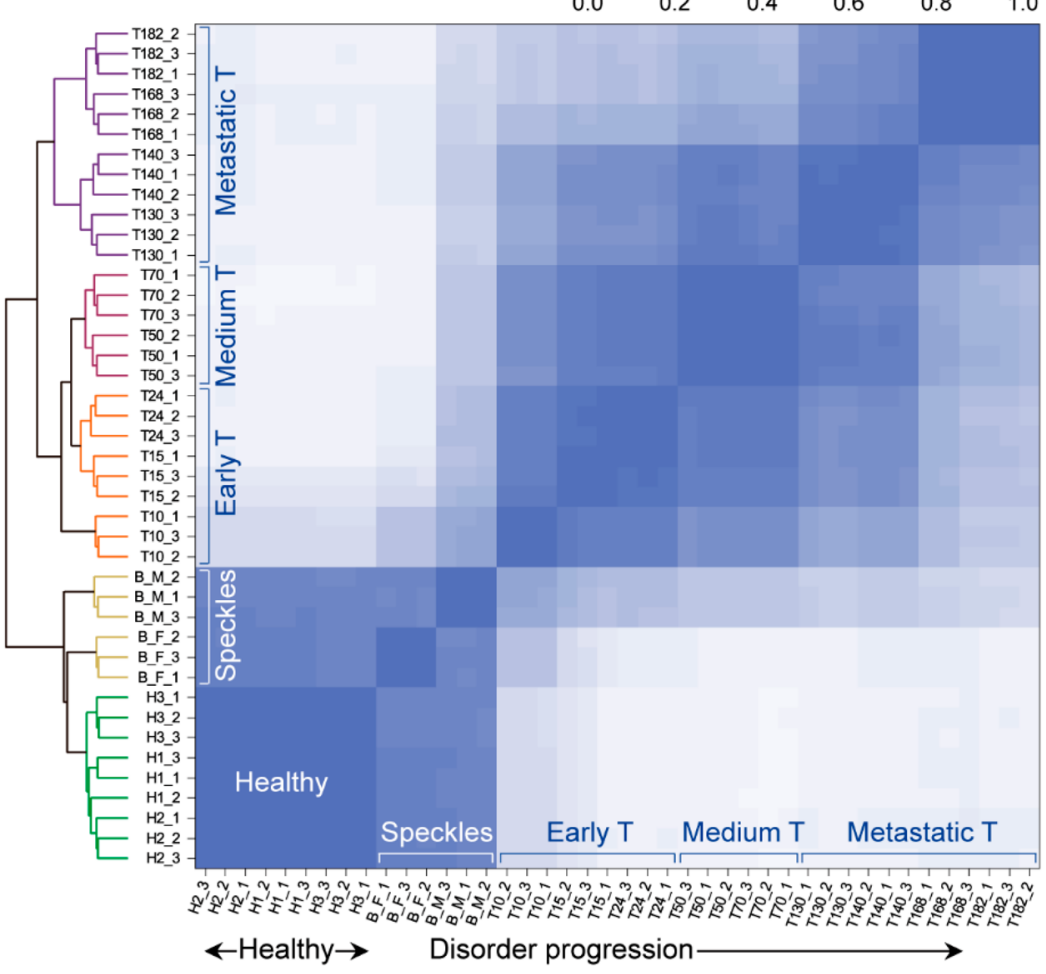

\begin{tabular}{|c|c|c|c|c|c|}
\hline 0.96 & 0.98 & 0.98 & 0.99 & - DecisionStump & D \\
\hline 0.96 & 0.98 & 0.98 & 0.99 & -SimpleCart & \\
\hline 0.96 & 0.98 & 0.98 & 0.99 & - J48graft & \\
\hline 0.96 & 0.98 & 0.98 & 0.99 & $-\mathrm{J} 48$ & $\mathbf{A}$ \\
\hline 0.96 & 0.98 & 0.98 & 0.99 & - Id3 & \\
\hline 0.96 & 0.98 & 0.98 & 0.99 & -BFTree & \\
\hline 0.96 & 0.98 & 0.98 & 0.99 & -LWL & \\
\hline 0.96 & 0.98 & 0.98 & 0.98 & -LogitBoost & \\
\hline 0.96 & 0.98 & 0.98 & 0.99 & -END & \\
\hline 96 & 0.98 & 0.98 & 0.99 & MultiB & \\
\hline
\end{tabular}
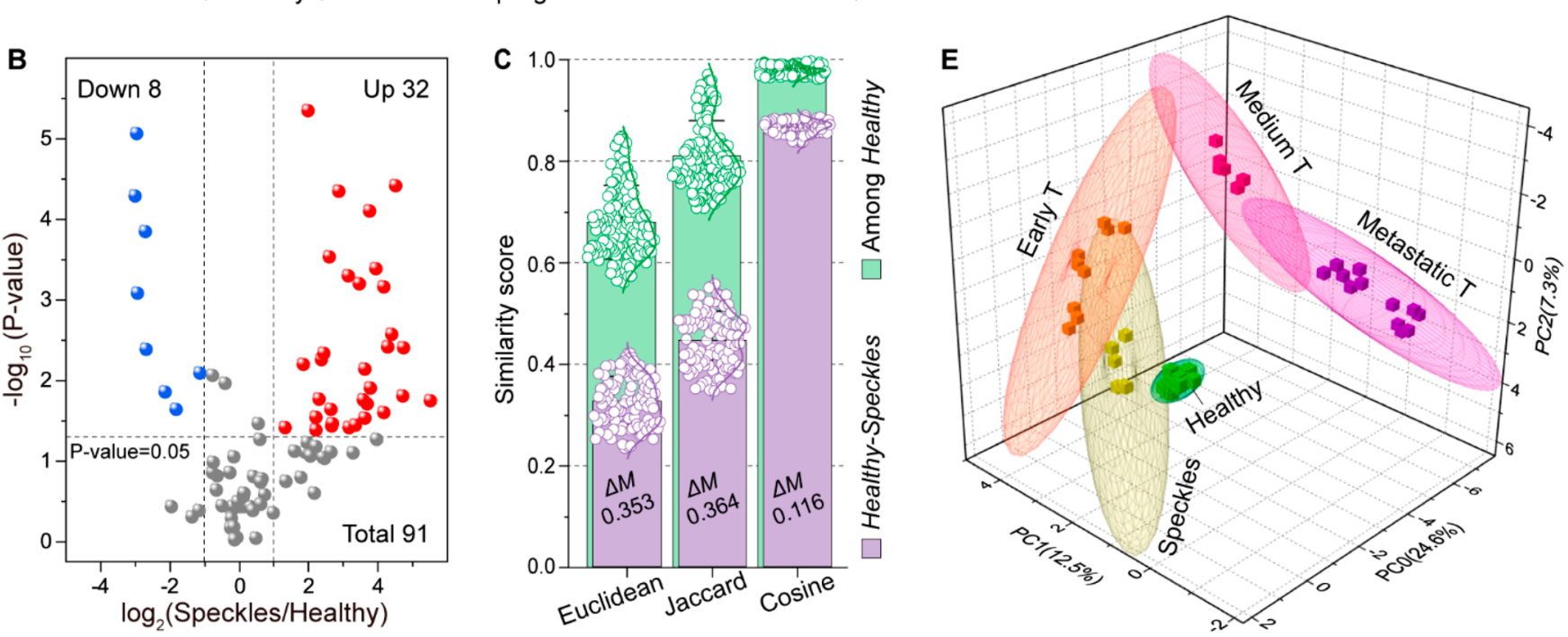

Figure 4. Data interpretation by analysis of the mass fingerprints on the whole. The skin disorder underwent the following progression stages: healthy state (Healthy), black speckle lesions (Speckles), early tumors (Early T), medium-sized tumors (Medium T), and metastatic tumors (Metastatic T). (A) Hierarchical clustering of the skin surface mass fingerprints using cosine similarity and average linkage. (B) Comparison of each peak relative intensity between Healthy and Speckles, with the $t$ test $P$ value plotted versus the magnitude of log 2-fold change. (C) Fingerprint similarity among Healthy, or between Healthy and Speckles, scored by the algorithm of relative Euclidean distance, Jaccard index, or cosine correlation (mean value bar, data dot distribution, mean difference $\Delta M$ among the two groups of data sets). (D) Performance of 28 machine learning algorithms for the detection of the malignant skin disorder, evaluated by Kappa statistic, accuracy, F-measure, and ROC area (weighted average value among classes). (E) Principal component analysis based on the first three principal components, with a 95\% confidence ellipsoid applied to each progression stage.

factors (here, the first three principal components PC0, PC1, and PC2). Due to the dimensionality reduction, it is possible to reveal small differences from the fingerprint peaks. PCA plot of the mass fingerprints from different progression stages is displayed in Figure 4E (PCA scores in Table S6), with the grouping result confirmed through a statistical analysis of the PCA scores by one-way analysis of variance (ANOVA) (Figure S14 and Table S7). It was observed that the $95 \%$ confidence ellipsoids of different tumor stages gradually separated along with the disorder progression. This indicates that the proposed platform could monitor skin disordering over time and predict the disorder progression stage through pattern matching of the fingerprints. Note that the PCA plot showed a partial overlap between the nearby stages of progression, for instance, between early tumors and medium-sized tumors or between medium-sized tumors and metastatic tumors. This could be 
A $\square$ Healthy $\square$ Early $T \square$ All tumors

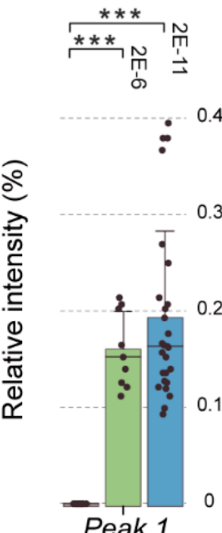

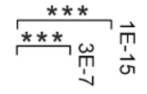

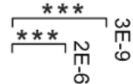

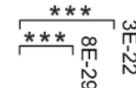
$\prod_{\substack{N \\ N}}^{\infty}$

Peak 1

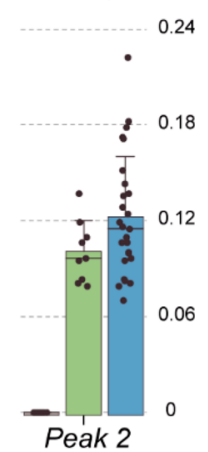

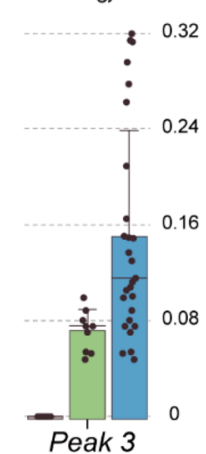

Peak 3

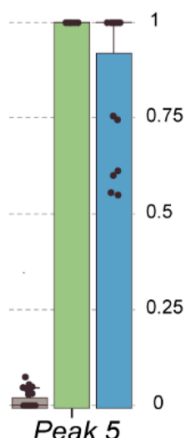

Peak 5

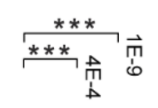

. 0.8

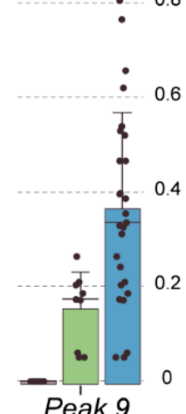

Peak 9

\section{D $\square$ Early T $\square$ Medium T $\square$ Metastatic T}
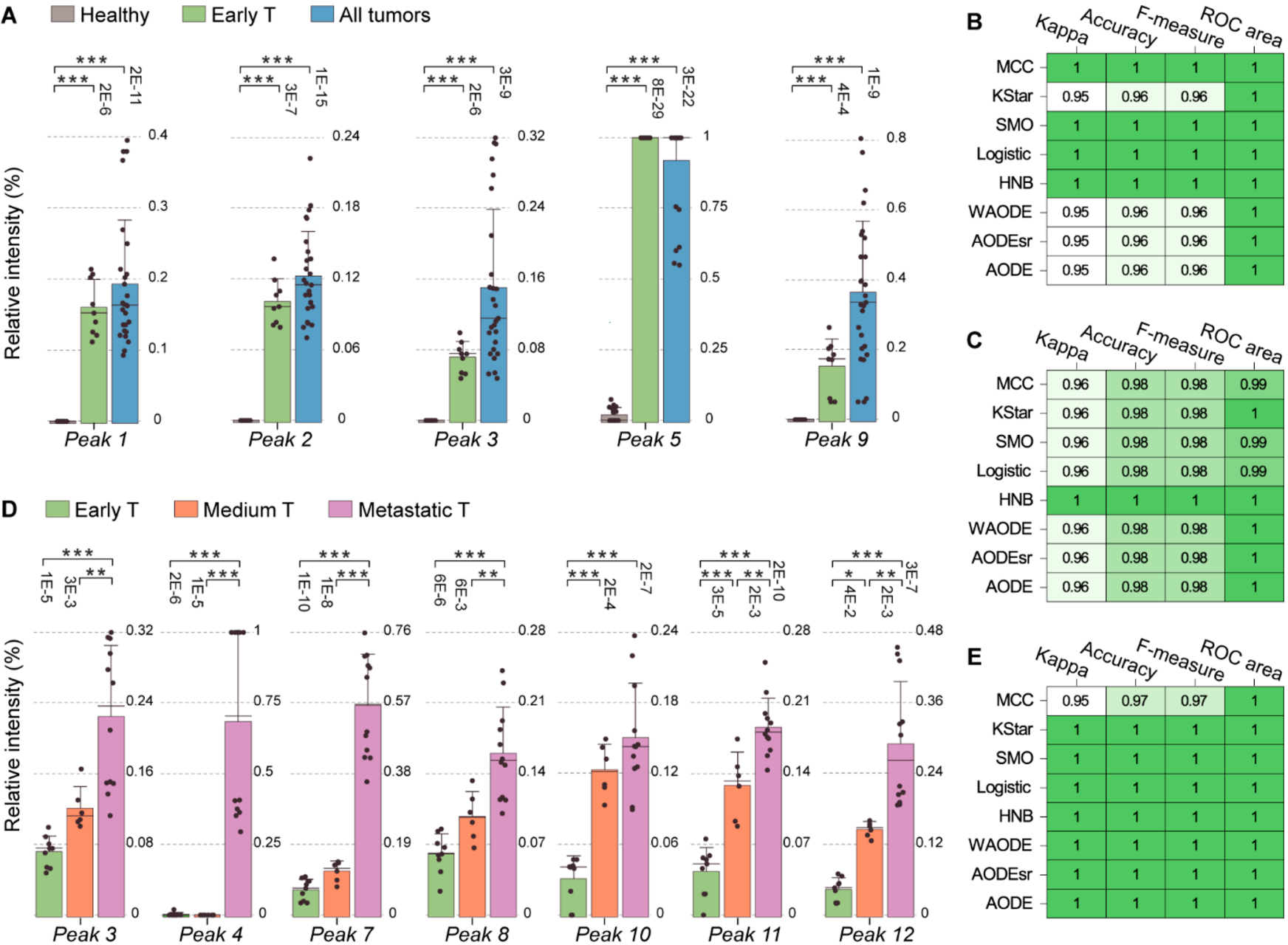

\section{E}

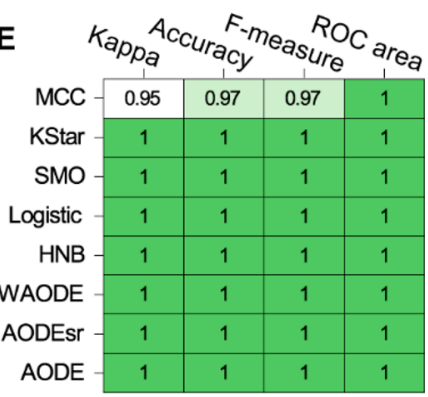

Figure 5. Data interpretation by tracking marker peaks. (A-C) Marker mass peaks helpful for skin disorder detection, with the relative peak intensity statistically compared among Healthy, Early $T$, and all tumors by one-way ANOVA (A); performance of these marker peaks for the classification between Healthy and Speckles (B) or between Healthy and all cancerous lesions (C), using machine learning classifiers. (D,E) Marker mass peaks useful to monitor the cancer progression, with the relative peak intensity statistically compared among Early T, Medium-sized T, and Metastatic $T$ by one-way ANOVA (D); performance of these marker peaks for the classification among cancer lesions at different progression stages (i.e., Speckles, Early $T$ and Medium-sized $T$, and Metastatic $T$ ) using machine learning classifiers (E). Star rating: $* P$ value $\leq 0.05$, $* * P$ value $\leq 0.01$, $* * * P$ value $\leq 0.001$. In $(\mathrm{B}, \mathrm{C}, \mathrm{E})$, machine learning algorithms with top performance in Figure $4 \mathrm{D}$ were selected for the classification. Classification performance measures: Kappa statistic, accuracy, F-measure, and ROC area.

explained by a gradual change of the molecular composition in the tumor microenvironment along with the disease progression. The variance in tumor growth dynamics among mouse individuals could also make an impact.

In addition to analysis of the fingerprint pattern on the whole, the building of marker mass peaks also facilitated the disorder detection and monitoring. To select peaks differentiating the cancerous from the healthy skins, Fisher's extract test was applied after a comparison of the fingerprint peak location, number and relative intensity (r.int.) (details in Figure S15). The top five most relevant peaks were found to be Peaks 1, 2, 3, 5, and 9 labeled previously in Figure 3. As statistically validated in Figure 5A, each of them was detected from all the tumor lesions, especially from all the early tumors, with a considerable intensity (r.int. $0.19 \pm 0.09,0.12 \pm 0.04$, $0.15 \pm 0.09,0.92 \pm 0.16,0.36 \pm 0.20$, respectively), but not detectable or detected with an extremely low intensity from healthy skins (r.int. $0,0,0,0.02 \pm 0.02,0$, respectively) ( $P$ values $\leq 0.001)$. When only these peaks were considered, the skin cancer was also detected with high confidence, especially at early stages, using machine learning models selected from Figure 4D (Figure 5B for the detection of speckle lesions, Figure 5C for all cancerous lesions). Similarly, some of the fingerprint peaks were found to be useful for monitoring the disorder progression. They were Peaks 3, 4, 7, 8, 10, 11, and 12 labeled in Figure 3. As statistically validated in Figure 5D, intensities of these peaks increased significantly ( $P$ values $\leq 0.05$ ) along with the tumor growth, and reached a considerable level at the metastatic stage (mostly more than quadrupled from early tumors). They allowed a confident classification among the cancer progression stages, as investigated with the same machine learning models used in Figure 5B,C (Figure 5E). By tracking the marker peaks, noting their presence, absence, or intensity changes, it is possible to make a quick prediction of the disorder occurrence, seriousness, and development trend.

Molecular identities of the above marker peaks were tentatively clarified through correlation with top-down proteomic data obtained from the excised skin tissues. The peak assignment is feasible as both MALDI-TOF and top- 

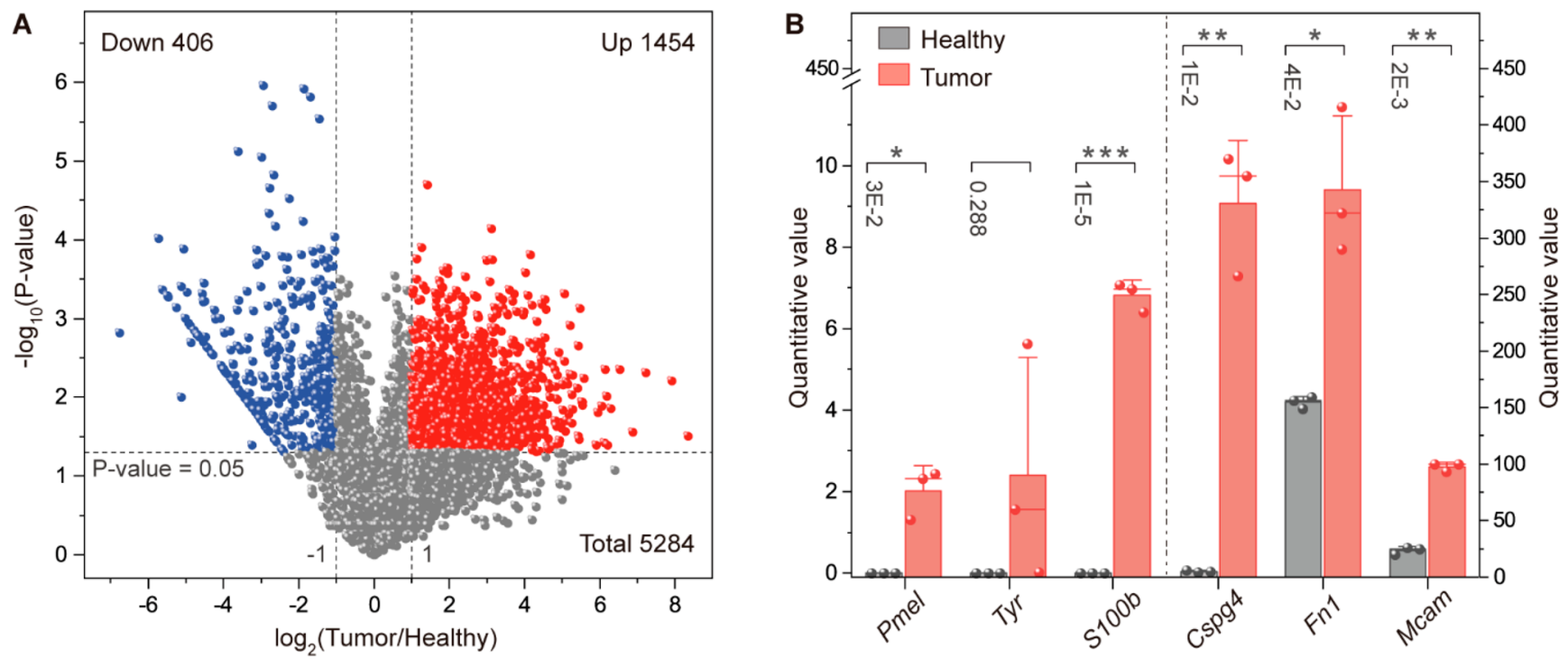

Figure 6. Result validation via quantitative proteomic analysis. Quantitative proteomics was performed with the excised mouse skin tissues using Scaffold proteome software. Three tumor lesions $\left(70,130\right.$, and $182 \mathrm{~mm}^{2}$ ) were analyzed as representatives, with the quantitative value (i.e., normalized total spectral count) of each identified protein statistically compared to the healthy controls. (A) Comparison of all the protein quantitative values between tumorous and healthy skins, with the paired $t$ test statistical significance $(P$ value $)$ plotted versus the magnitude of log 2 -fold change. (B) Comparison of quantitative values from six well-established melanoma biomarkers between tumorous and healthy skins (mean value bars, median value lines, data dot distribution; star rating: $* P$ value $\leq 0.05$, ** $P$ value $\leq 0.01$, ***P value $\leq 0.001)$.

down proteomics measure intact proteins with the signal intensity highly related to the protein abundance. ${ }^{28}$ Accordingly, Peak 1 could be assigned to thymosin $\beta$-4, an actin monomer-binding protein impacting cell mobility by regulating the cell adhesion. ${ }^{29}$ Peak 2 possibly represents vimentin, the only type of intermediate filament contained by melanoma cells to maintain the cell integrity and a suggested biomarker for melanoma diagnosis. ${ }^{30}$ Peak 3 could originate from LINE-1 retrotransposable element ORF1 protein, whose expression is a hallmark of many types of malignancy. ${ }^{31}$ Peaks 4 and 10 might be from actin $\beta$ and actin $\gamma$ cytoplasmic 1, highly expressed in skin cancers to regulate the cell proliferation, motility, and migration. ${ }^{32}$ Peak 5 is likely from ATPase inhibitory factor 1 , overexpressed to promote cancer cell survival under temporary anoxic conditions possibly by preserving cellular ATP despite mitochondrial dysfunction. ${ }^{33}$ The S100 calcium binding proteins including S100a8 (Peak 7) and S100b (Peak 8) are involved in many phenotypic features of cancer cells and are widely used as skin cancer diagnosis markers. ${ }^{34}$ Peak 9 is possibly from heat shock protein 1, released from malignant cells to the extracellular space to form a fostering environment beneficial for the tumor growth. ${ }^{35}$ Peaks 11 and 12 could be from hemoglobin subunit $\alpha$ and $\beta$-2, whose level in solid tumors can be influenced by the hypoxic tumor microenvironment due to aberrant vascularization and poor blood supply. ${ }^{36}$ Peak assignment information was given in Data File S1.

As a validation of the above detection results, quantitative proteomics was conducted with the excised skin tissues. ${ }^{37}$ Compared to the healthy controls, the tumor lesions had more than $30 \%$ of proteins differently expressed, including $\sim 28 \%$ upregulated and $\sim 8 \%$ down-regulated (Figure $6 \mathrm{~A}$ by spectral counting, Figure S16A by label-free quantification, more details in Data File S2). It clearly showed that the skin protein composition was significantly changed when the disorder happened. This coincided with changes of the skin surface mass fingerprints, demonstrating the capability of the new method for skin state investigations at the molecular level.
Among the up-regulated proteins, we found six biomarkers widely used for the diagnosis and prognosis of melanoma. They were premelanosome protein (Pmel), tyrosinase (Tyr), S100 calcium binding protein B $(S 100 b)$, chondroitin sulfate proteoglycan 4 (Cspg4), fibronectin 1 (Fn1), and melanoma cell adhesion molecule (Mcam) (Figure 6B, Figure S16B, and Data File S3). ${ }^{38}$ This confirmed the investigated tumor lesions as melanoma. Notably, among these biomarkers, $S 100 \mathrm{~b}$ was also observed on the skin surface mass fingerprints as Peak 8 $(10,598 \mathrm{~m} / \mathrm{z})$, exclusively from the tumor lesions not the healthy controls, further showing the feasibility of the new methodology. The other biomarkers were not observed on the fingerprints as they were large proteins exceeding the 2000$20,000 \mathrm{~m} / z$ mass recording window.

\section{Extension Test on Adjacent Nondisorder Skin on Mice}

Along with the tumor growth, mass fingerprints of the adjacent nontumor skin regions (located $\sim 15 \mathrm{~mm}$ away from the tumor lesions) were also found to be changed. At early stages with tumor size smaller than $70 \mathrm{~mm}^{2}$, the adjacent nontumor skin mass fingerprints resembled the healthy controls with cosine similarity higher than 0.900 (representatives in Figure 7A, Healthy, T50, T70). The skin looked like that in a healthy state, displaying similar color and texture. However, the skins were found slightly bluish and stiffer when the tumor started metastasis, and the mass fingerprints were different from the healthy ones with a great decline of the dominant peak at 9979 $m / z$ (representatives in Figure 7A, T130, T168, T182). The fingerprint difference could be explained by the collection of fewer epidermal cells due to the increase of skin stiffness. During the tumor growth, the cancer cells produced a high content of melanin pigment, which could be obtained by the surrounding skin cells through intercellular transfer and cell fusion. ${ }^{39}$ Intracellularly, the melanin is present in the form of microsized granules, i.e., melanosomes, which are quite hard to deform. ${ }^{40}$ The accumulation of melanosomes thus led to the increase of stiffness and the mildly bluish color observed on the 


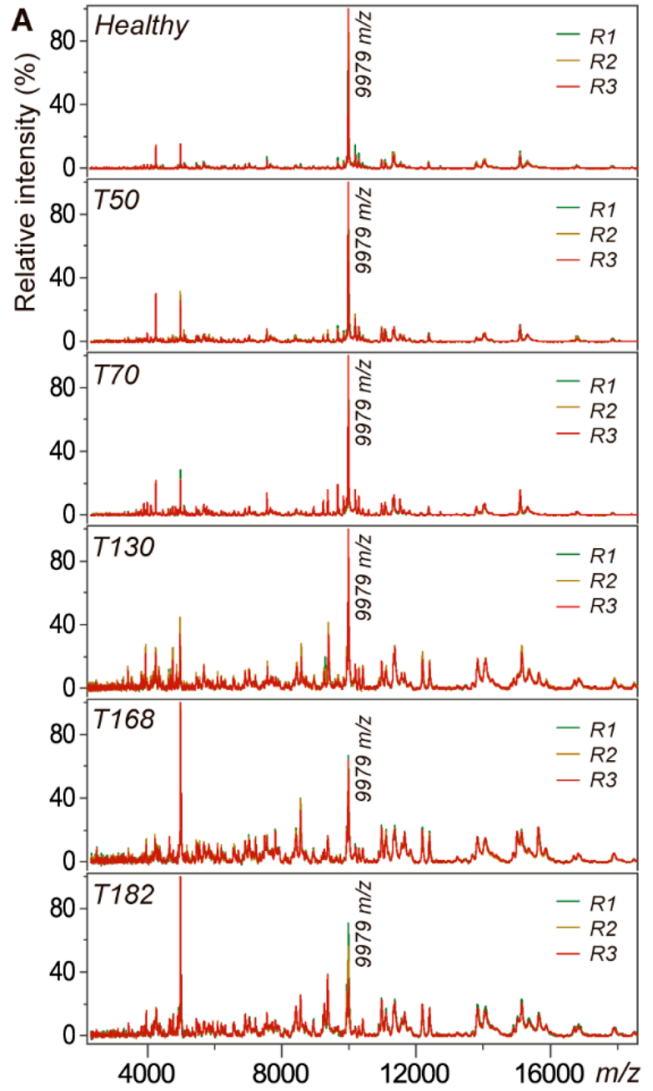

B

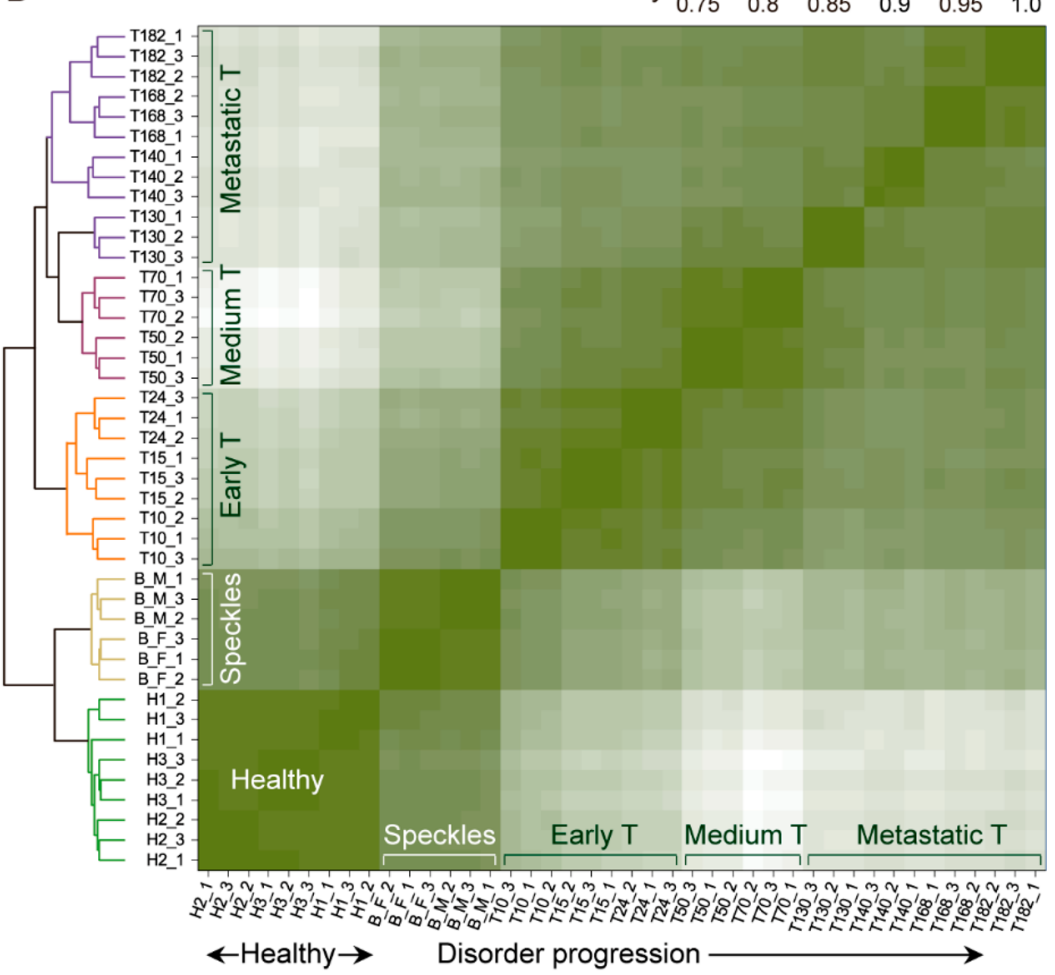

D

New approach $\square$ Blood test $\square$

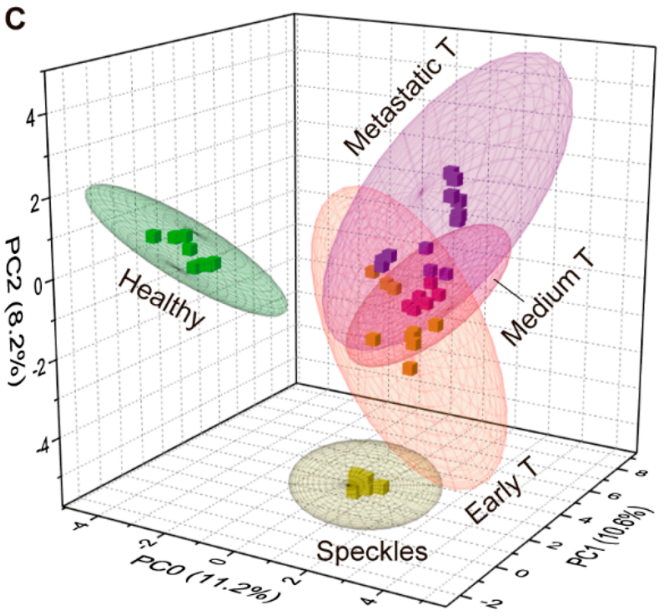

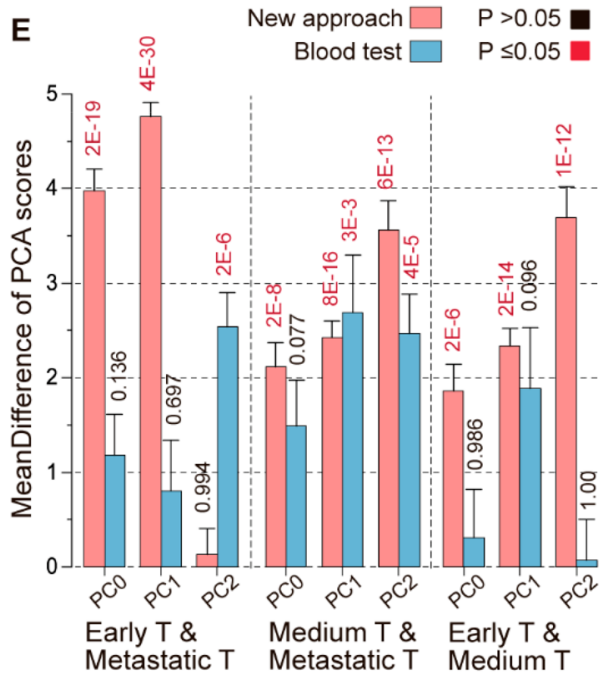

Figure 7. Extension tests on adjacent skins and comparison test with blood. (A) Mass fingerprints from adjacent nontumor skins (located around $15 \mathrm{~mm}$ away from the tumor lesions) when the tumor size was 50, 70, 130, 168, or $182 \mathrm{~mm}^{2}$. (B,C) Analysis of the skin malignancy by a blood test, i.e., detection of blood-circulating exosomes using MALDI-TOF mass spectrometry: (B) hierarchical clustering based on spectral cosine similarity and average linkage and (C) principal component analysis by the first three principal components with $95 \%$ confidence ellipsoids applied to the malignancy progression stages. (D,E) Comparison of the blood test and the new methodology for the differentiation among the disorder progression stages, (D) by scoring spectral cosine similarities (mean value bar and label, median value line, data dot distribution) or (E) by statistical analysis of the principal component scores (mean difference bar, $P$ value $\leq 0.05$ for significant difference).

adjacent regions. The increased stiffness could reduce cell adhesion tendency to the sampling discs, and therefore, fewer cells were collected (microscopic images in Figure S17). This phenomenon might also be used as a sign to indicate the cancer metastasis. A deeper investigation was not included here. Notably, although different from healthy controls, the adjacent nontumor skin fingerprints remained distinct from the tumor lesions with cosine similarity lower than 0.200 (Figure S18).

\section{Comparison Test with Blood in Mice}

The relevance of this new methodology for the detection and monitoring of skin disorders was further demonstrated through comparison with a blood test. Blood testing is frequently involved in clinics due to the rich bioinformation in blood and the easy sampling procedure. For the study of malignancy like cancer, blood-circulating exosomes are the "hot" target analytes. $^{41}$ They participate in malignancy progression in a variety of ways and are not difficult to isolate. ${ }^{42}$ Here, the 
blood exosomes derived throughout the skin cancer growth were analyzed by MALDI-TOF MS to correlate with the detection results from the new methodology. Along with the cancer growth, the exosomal mass fingerprints also changed gradually (Figure S19), with the different mass peaks mostly coming from commonly used blood biomarkers for malignancy prediction, like complement component 3, fibrinogen $\alpha$ chain, haptoglobin, serum amyloid A, and hemoglobin subunits (Data File S4). ${ }^{43,44}$ The differentiation results between cancerous and healthy mice according to the exosomal fingerprints, through either HCA (Figure 7B, cluster distance in Figure S20, cosine similarities in Table S8) or PCA (Figure 7C, scores in Table S9), were generally consistent with the results from the new methodology. This once again validated the reliability of the new method. Comparing the two approaches, the new method was found more performant for the differentiation between different stages of the cancer progression. It generated mutual cosine similarity of $0.601( \pm 0.147), 0.721( \pm 0.162)$, and $0.862( \pm 0.063)$ among the early tumors, medium-sized tumors, and metastatic tumors, whereas the mutual similarity from the blood test was much higher, being $0.942( \pm 0.008)$, $0.957( \pm 0.007)$, and $0.964( \pm 0.009)$, respectively (Figure $7 \mathrm{D})$. This was also indicated by the PCA plot, as the new method produced less overlapping of the $95 \%$ confidence ellipsoids (Figure 4E versus Figure 7C) and larger statistical difference among the principal component scores (Figure 7E, Figure S14 versus Figure S21, Table S7 versus Table S10) among the tumor stages. Considering the analyte location, a large proportion of blood background components produced by various types of body cells could suppress the signals from the skin disorder and thus limit the blood test sensitivity. The new method directly investigated the skin, where the disorder arose, and therefore provided much more specific bioinformation to assist the disorder analysis. This argumentation should also hold true for other types of skin malignancy.

\section{DISCUSSION}

A methodology for skin monitoring has been developed here, through the coordination of skin surface adhesive sampling, MALDI-TOF mass recording, and algorithm-directed data interpretation. As demonstrated above, the methodology allows a routine investigation of skin state, an early detection of skin disorder, and a dynamic monitoring of the disorder progression. Compared to existing skin analysis strategies, this new approach shows advantages in five aspects: (i) noninvasive due to the mild adhesive sampling, (ii) time-saving with each step taking only a few minutes, (iii) high-throughput owing to the hundred-spot mass spectrometer target, (iv) highly sensitive with skin malignancy detectable as early as the occurrence of submillimeter-sized lesions, and (v) allowing automated data interpretation to achieve high accurate analysis, e.g., detection of the skin malignancy with $100 \%$ accuracy.

Regarding the sampling methods in the dermatological study, skin biopsy is the state-of-the-art procedure; however, it is often painful, risky, and limited to the surgery scheduling. Whenever allowed by a dermatologist, the noninvasive adhesive sampling can partially replace skin biopsy to provide a patient-friendly diagnosis. In order to provide a reproducible detection, the sampling protocol should be kept consistent, as parameters like the materials of sampling discs and the sampling repetition times could influence the quality of the collected skin samples. Researchers have made continuous efforts to make the process user-friendly, with the distribution of commercial kits in high quality and low price, like the kits from DermTech and D-Squame (used here).

The MALDI-TOF mass fingerprinting stands out from other analytical strategies due to its speed and simplicity. It can analyze a large number of samples in a short time without complicated experimental operations. It is readily compatible with the adhesive sampling, where the collected skin samples can be analyzed straightforwardly without any sample pretreatment. This label-free measurement helps the collection of sample information on the whole without bias. The mass fingerprinting procedure can also be coupled with mass spectrometry imaging to investigate the skin from more aspects, for instance, description of spatial distribution of diagnosis-relevant molecules or detection of the skin lesion boundaries.

By algorithm-directed data analysis, the methodology achieved a quick and accurate result interpretation. For the scoring of mass fingerprint similarity, we employed here mostly the algorithm of cosine correlation, which considers both the peak presence and intensity. It generally provided satisfactory detection results for the skin cancer at various stages of progression. To explore minor intersample differences, for instance, study of very early skin disorder with minor abnormality or differentiation between nearby stages of disorder progression, an algorithm with increased weighting of peak presence and decreased weighting of peak intensity could generate better results. Jaccard index and Euclidean distance were proposed as such measures (Figures S12, S13, and S22). Other often-used measures like Pearson's correlation were not investigated here, but they might be options. The machine learning models used here were extracted from the Weka algorithm library. For the mouse skin cancer, the learning models listed in Figure 5 performed best with the new method. A deeper comparison of currently available learning models using much larger skin disorder data sets is going to be made in the future to select the most appropriate one or combination to reach the most reliable data interpretation. A dedicated algorithm could also be realized to reach an easy tracking of disease-characteristic peaks on the fingerprints, following an example recently reported for the analysis of liquid chromatography data. ${ }^{45}$ On the whole, the algorithmdirected data learning helps to reach automated result interpretation, reducing the heavy reliance on skilled physicians with prior clinical experience.

One more concern is about the protein assignment of the mass fingerprint peaks. We made the assignment through correlation with top-down proteomic data by matching the peak mass with the detected protein mass. This procedure is much more reliable than directly matching the peak mass with the theoretical protein molecular weight provided by databases like UniProt. A protein detected from a biological sample is not always in its theoretical full length as recorded in databases, due to a variety of cellular processes and possibly experimental operations. The assignment outcome matched well with a previous proteome study on melanoma tissue biopsies, showing the rationality of this procedure. ${ }^{46}$ Nevertheless, this assignment is still at a tentative level, as the sample preparation and measurement process of MALDI-TOF mass fingerprinting are different from the proteomic analysis. A more precise procedure might be using high-resolution MALDI tandem mass spectrometry to conduct in situ bottom-up proteomics. 
In this work, melanoma was used as a skin disease model. In clinics, it is often an important task to differentiate between early melanoma and skin moles, due to their similarity in physical appearance. The differentiation is, in principle, possible using the new methodology, as the protein profile of melanoma cells were considerably different from the melanocytes forming the moles. ${ }^{47}$ This is also indicated by a preliminary comparison test between human melanoma cells and skin moles (Figure S23). Generally, it is promising to apply the new methodology to a broad range of skin conditions, including other types of skin cancer like mycosis fungoides, basal cell carcinoma, and squamous cell carcinoma, as well as skin diseases with different pathogenesis like wound (i.e., burn), allergy (i.e., atopic dermatitis), infection (i.e., leprosy, chancroid), keratinocyte hyperproliferation (i.e., psoriasis), and so on. This is supported by a recent finding that the skin molecular composition is specific to the disease type and pathogenesis, after investigation of 311 skin biopsy samples related to 16 common types of skin diseases. ${ }^{48}$ It has also been reported that the molecular signature is changeable along with the disease progression or treatment. ${ }^{49}$ This indicates that the methodology we developed could be used widely for skin analysis, helpful to explore physiological and pathological aspects of various skin states.

The implementation of this methodology in practice could be facilitated by building a comprehensive skin surface mass fingerprint database from common skin conditions. In order to reach this goal, we are currently applying for a test permission on human patients through the collaboration with local dermatologists. The database could be integrated with proper similarity measures, machine learning tools, and peak tracking algorithms to achieve a straightforward data analysis on a single software platform. The construction of such a database could be labor-intensive, but it will greatly promote the result interpretation once completed. Currently, MALDI-TOF mass spectrometers have been widely installed in clinical laboratories, opening doors for a routine application of this methodology. The rapid, high-throughput, and patient-friendly detection manner also provides the opportunity to build personalized skin care in the future.

\section{MATERIALS AND METHODS}

\section{Ethics Statement}

The studies on mice were performed in accordance with Swiss federal regulations and procedures approved by veterinary authority of Canton Vaud. D-Squame brand adhesive sampling discs employed in this work have passed human skin safety tests and obtained the European Certification (CE mark). Samples from healthy human skin surfaces were subjected to MALDI-TOF MS measurements only, without manipulation of the genetic materials. All biological wastes were deposited properly according to the biosafety rules issued by École Polytechnique Fédérale de Lausanne.

\section{Development of Mouse Model Skin Cancer}

The skin cancer was induced using genetically engineered method, by treating Tyr::CreER;BRaf ${ }^{C A}$; ten $^{\text {lox4-5 }}$ transgenic mice with 4-hydroxytamoxifen (4-HT) topically on the back skin. This cancer development procedure has been described previously by Dankort et al. in detail. ${ }^{21,22}$ The mice harbored a 4-HT-inducible Cre recombinase-estrogen receptor fusion transgene (CreER) under the control of melanocyte-specific tyrosinase (Tyr) promoter. The mice also carried conditional alleles of BRaf $\left(B R a f^{C A}\right)$ and Pten $\left(\right.$ Pten $\left.^{\text {lox4-5 }}\right)$. First, $1.5 \mu \mathrm{L}$ of 4 -HT ( $50 \mathrm{mg} \cdot \mathrm{mL}^{-1}$ in dimethyl sulfoxide) was applied topically on the back skin of the mice at 3 weeks old, using a small paint brush. Activation of CreER by 4-HT led to the melanocyte- specific expression of BRAF V600E and the silencing of PTEN. The $B R A F$ V600E mutation is the most common genetic alteration in human melanoma, and the silencing of tumor suppressor gene PTEN promotes malignant progression. By controlling the tumor growth time, the mice were developed with melanoma at different progression stages.

\section{Skin Sampling}

On healthy human volunteers, the skin regions to be sampled were washed with soap, rinsed with running water, and left to dry naturally. D-Squame adhesive sampling discs were used for the sampling. The disc was composed of transparent polyester film and an acrylic adhesive layer. The adhesive side was attached to a suspicious skin region. A mild lateral pressure was applied for $10 \mathrm{~s}$ to achieve a good adhesion between the adhesive layer and the skin surface. The boundary of the suspicious region was outlined on the polyester film using a watercolor pen. The disc was then stripped off the skin surface by holding one corner. The sampling on mice was performed by a similar procedure, after removing the skin hair carefully using a razor blade and cleaning the skin surface gently using a wipe containing $70 \%$ ethanol. Each skin region was sampled multiple times in a row (e.g., four times), using a new disc each time. The multiple samplings helped to ensure reliability of the detection result. The obtained samples were either analyzed immediately or stored in $4{ }^{\circ} \mathrm{C}$ for later analysis within $24 \mathrm{~h}$. For mass spectrometry measurements, the sampling discs carrying collected epidermis cells were attached to the MALDI target using a Scotch double-sided tape (3M Science, USA), with the cell-collection side facing up. The MALDI matrix was pipetted to cover the outlined sample region and left to dry at room temperature to form cell-matrix cocrystals. The target was then loaded into mass spectrometer for measurement.

\section{MALDI-TOF MS Measurements}

Sinapinic acid, $15 \mathrm{mg} \cdot \mathrm{mL}^{-1}$ in 50/49.9/0.1\% (volume percentage) acetonitrile/water/trifluoroacetic acid, was used as the MALDI matrix. All measurements were conducted on Bruker microflex LRF MALDI-TOF mass spectrometer under linear positive mode with delayed extraction. The positive mode benefits the detection of cellular proteins as they have high proton affinities with the tendency to be ionized through protonation. ${ }^{13}$ The delayed extraction involves a time delay ( $400 \mathrm{~ns}$ here) between the laser pulse and the ionaccelerating voltage, providing time-of-flight compensation for ion velocity spread to improve mass resolution. ${ }^{50}$ The instrumental parameters were set as laser intensity $70 \%$, laser attenuator with $30 \%$ offset and $40 \%$ range, laser frequency $20.0 \mathrm{~Hz}$, detector gain $20 \times$, suppress up to $1000 \mathrm{Da}$, mass window $2000-20,000 \mathrm{~m} / z$. The mass window lower than $2000 \mathrm{~m} / z$ suffered from interference from the matrix, and the window higher than $20,000 \mathrm{~m} / z$ had the distribution of only low-abundant peaks due to the low ionization efficiency of large proteins from the whole intact cells. Mass calibration was conducted with an aqueous solution containing cytochrome $c$, myoglobin, and protein $\mathrm{A}\left(1 \mathrm{mg} \cdot \mathrm{mL}^{-1}\right.$ for each). For each skin sample, the mass spectrum was obtained with $5 \times 250$ laser shots throughout the sample region to reduce the possible impact from "sweet" spots and to generate a panorama of the whole skin region.

\section{Protein Assignment for MALDI-TOF MS Peaks}

Skin surface mass fingerprint peaks were tentatively assigned to proteins by mass matching with the skin tissue top-down proteomic data. Most ions detected by MALDI-TOF MS were singly charged, and thus the protein mass was $\left[(\mathrm{m} / z)_{\text {peak top }}-1\right]$ under linear positive detection mode. This mass corresponded to the molecular average mass, obtained by summing the average atomic mass of each constituent element. For each protein identified by top-down proteomics, the average mass was calculated according to the measured protein sequence, using a peptide mass calculator like PeptideSynthetics. In this regard, the average mass measured by MALDI-TOF MS was correlated with the average mass obtained from top-down proteomics. Considering the mass resolution of liner TOF, $1000 \mathrm{ppm}$ tolerance was allowed for the mass matching. If one MALDI-TOF MS peak could be correlated with multiple proteins in 
the top-down proteomic data according to the mass value, the peak was then assigned to the protein detected with the highest number of matching fragments with an $E$ value (expectation value) lower than 0.0001 , i.e., the most abundant protein detected with high confidence around that mass.

\section{Data Analysis}

The MALDI-TOF mass spectra were processed using Mass-Up software for smoothing (moving average), baseline correction (Snip algorithm), peak picking (MALDIquant algorithm, signal-to-noise ratio 3.0, half-window size 60), and peak alignment (Forward algorithm, $1000 \mathrm{ppm}$ mass tolerance). ${ }^{51}$ Data analysis by machine learning, including the unsupervised learning of HCA, PCA, and the supervised learning of classification, was conducted in the Weka machine learning environment implanted on the Mass-Up platform. Mass spectral similarity scoring was conducted on the BacteriaMS software platform, using the algorithm of Jaccard index, relative Euclidean distance, intensity-weighted Euclidean distance, or cosine correlation. These algorithms were explained in the Algorithm Description section in the Supporting Information. All of the statistical analyses were conducted via a hypothesis $t$ test (for two groups of data) or ANOVA (for three or more groups of data) using the OriginLab software, with the statistical significance conditioned by a $P$ value $\leq 0.05$.

\section{ASSOCIATED CONTENT}

\section{Supporting Information}

The Supporting Information is available free of charge at https://pubs.acs.org/doi/10.1021/jacsau.0c00074. Raw data from the proteomics and mass spectrometry are available at https://data.mendeley.com/datasets/vrknzdvv8b/draft? $a=$ 4ccbfe98-b296-4f67-bb14-d99cddfd2c50 from the Mendeley Data set.

Supplementary methods, algorithm description, supplementary figures (Figures S1-S23), supplementary tables (Tables S1-S10), supplementary data files (data files $\mathrm{S} 1-\mathrm{S} 4)$ (PDF)

\section{AUTHOR INFORMATION}

\section{Corresponding Author}

Hubert H. Girault - Institute of Chemical Sciences and Engineering, School of Basic Sciences, Ecole Polytechnique Fédérale de Lausanne, 1015 Lausanne, Switzerland; ○ orcid.org/0000-0001-5573-5774;

Email: hubert.girault@epfl.ch

\section{Authors}

Yingdi Zhu - Institute of Chemical Sciences and Engineering, School of Basic Sciences, Ecole Polytechnique Fédérale de Lausanne, 1015 Lausanne, Switzerland

Andreas Lesch - Department of Industrial Chemistry "Toso Montanari”, Universita degli Studi di Bologna, 40136 Bologna, Italy; ○ orcid.org/0000-0002-4995-2251

Xiaoyun Li - Department of Fundamental Oncology and Ludwig Institute for Cancer Research, Université de Lausanne, 1066 Epalinges, Switzerland

Tzu-En Lin - Institute of Biomedical Engineering, College of Electrical and Computer Engineering, National Chiao Tung University, 30010 Hsinchu, Taiwan

Natalia Gasilova - Institute of Chemical Sciences and Engineering, School of Basic Sciences, Ecole Polytechnique Fédérale de Lausanne, 1015 Lausanne, Switzerland
Milica Jovic - Institute of Chemical Sciences and Engineering School of Basic Sciences, Ecole Polytechnique Fédérale de Lausanne, 1015 Lausanne, Switzerland

Horst Matthias Pick - Environmental Engineering Institute, School of Architecture, Civil and Environmental Engineering, Ecole Polytechnique Fédérale de Lausanne, 1015 Lausanne, Switzerland

Ping-Chih Ho - Department of Fundamental Oncology and Ludwig Institute for Cancer Research, Université de Lausanne, 1066 Epalinges, Switzerland

Complete contact information is available at: https://pubs.acs.org/10.1021/jacsau.0c00074

\section{Author Contributions}

The manuscript was written through contributions of all authors. All authors have given approval to the final version of the manuscript.

Notes

The authors declare no competing financial interest.

\section{ACKNOWLEDGMENTS}

We gratefully acknowledge the Laboratory of Mass Spectrometry Analytical Chemistry in Fudan University for providing a free-access online software platform BacteriaMS for the mass spectrometry data processing. We also would like to thank the Proteomics Core Facility in École Polytechnique Fédérale de Lausanne for the conduction of quantitative bottom-up proteomic analysis. P.C.H. is supported in part by the SNSF Project Grants (31003A 182470), the European Research Council Starting Grant (802773-MitoGuide), and the EMBO Young Investigator Award. T.E.L. thanks the Young Scholar Fellowship Program by Ministry of Science and Technology (MOST) in Taiwan, under Grant MOST 108-2636-E-009-012.

\section{REFERENCES}

(1) Karimkhani Aksut, C.; Dellavalle, R.P.; Naghavi, M. Global skin disease morbidity and mortality: An update from the Global Burden of Disease Study 2013. J. Invest. Dermatol. 2017, 137 (5), S31-S31.

(2) Someya, T.; Amagai, M. Toward a new generation of smart skins. Nat. Biotechnol. 2019, 37 (4), 382-388.

(3) Joob, B.; Wiwanitkit, V. COVID-19 can present with a rash and be mistaken for dengue. J. Am. Acad. Dermatol. 2020, 82 (5), E177E177.

(4) Apalla, Z.; Nashan, D.; Weller, R. B.; Castellsague, X. Skin cancer: epidemiology, disease burden, pathophysiology, diagnosis, and therapeutic approaches. Dermatol Ther 2017, 7 (1), 5-19.

(5) Murphy, M. J. Molecular Diagnostics in Dermatology and Dermatopathology; Springer Science \& Business Media: New York, 2011.

(6) Fischer, A. H.; Jacobson, K. A.; Rose, J.; Zeller, R. Hematoxylin and Eosin Staining of Tissue and Cell Sections. Basic Methods in Microscopy; Cold Spring Harbor Laboratory Press, 2014.

(7) Dinnes, J.; di Ruffano, L. F.; Takwoingi, Y.; Cheung, S. T.; Nathan, P.; Matin, R. N.; Chuchu, N.; Chan, S. A.; Durack, A.; Bayliss, S. E. Ultrasound, CT, MRI, or PET-CT for staging and restaging of adults with cutaneous melanoma. Cochrane Database Syst. Rev. 2019, 7, CD012806.

(8) Margulis, K.; Chiou, A. S.; Aasi, S. Z.; Tibshirani, R. J.; Tang, J. Y.; Zare, R. N. Distinguishing malignant from benign microscopic skin lesions using desorption electrospray ionization mass spectrometry imaging. Proc. Natl. Acad. Sci. U. S. A. 2018, 115 (25), 6347-6352.

(9) Lin, T. E.; Bondarenko, A.; Lesch, A.; Pick, H.; Cortes-Salazar, F.; Girault, H. H. Monitoring Tyrosinase Expression in Nonmetastatic and Metastatic Melanoma Tissues by Scanning Electro- 
chemical Microscopy. Angew. Chem., Int. Ed. 2016, 55 (11), 38133816.

(10) Maedler, C.; Kim, D.; Spanjaard, R. A.; Hong, M.; Erramilli, S.; Mohanty, P. Sensing of the Melanoma Biomarker TROY Using Silicon Nanowire Field-Effect Transistors. Acs Sensors 2016, 1 (6), 696-701.

(11) He, H.; Bissonnette, R.; Wu, J.; Diaz, A.; Proulx, E. S.-C.; Maari, C.; Jack, C.; Louis, M.; Estrada, Y.; Krueger, J. G. Tape strips detect distinct immune and barrier profiles in atopic dermatitis and psoriasis. J. Allergy Clin Immun 2020, 147, 199-212.

(12) Darvishi, S.; Pick, H.; Lin, T. E.; Zhu, Y. D.; Li, X. Y.; Ho, P. C.; Girault, H. H.; Lesch, A. Tape-Stripping Electrochemical Detection of Melanoma. Anal. Chem. 2019, 91 (20), 12900-12908.

(13) Knochenmuss, R.; Zenobi, R. MALDI Ionization: The Role of In-Plume Processes. Chem. Rev. 2003, 103 (2), 441-452.

(14) Jaskolla, T. W.; Karas, M. Compelling Evidence for Lucky Survivor and Gas Phase Protonation: The Unified MALDI Analyte Protonation Mechanism. J. Am. Soc. Mass Spectrom. 2011, 22 (6), 976-988.

(15) He, J.; Baxter, S. L.; Xu, J.; Xu, J.; Zhou, X.; Zhang, K. The practical implementation of artificial intelligence technologies in medicine. Nat. Med. 2019, 25 (1), 30-36.

(16) Abonnenc, M.; Qiao, L. A.; Liu, B. H.; Girault, H. H. Electrochemical Aspects of Electrospray and Laser Desorption/ Ionization for Mass Spectrometry. Annu. Rev. Anal. Chem. 2010, 3, 231-254.

(17) Sandby-Møller, J.; Poulsen, T.; Wulf, H. C. Epidermal thickness at different body sites: Relationship to age, gender, pigmentation, blood content, skin type and smoking habits. Acta Derm-Venereol 2003, 83 (6), 410-413.

(18) Hoath, S. B.; Leahy, D. G. The organization of human epidermis: Functional epidermal units and phi proportionality. $J$. Invest. Dermatol. 2003, 121 (6), 1440-1446.

(19) Sprenger, A.; Weber, S.; Zarai, M.; Engelke, R.; Nascimento, J. M.; Gretzmeier, C.; Hilpert, M.; Boerries, M.; Has, C.; Busch, H.; Bruckner-Tuderman, L.; Dengjel, J. Consistency of the Proteome in Primary Human Keratinocytes With Respect to Gender, Age, and Skin Localization. Mol. Cell Proteomics 2013, 12 (9), 2509-2521.

(20) Sun, C.; Wang, L. Q.; Huang, S. D.; Heynen, G. J. J. E.; Prahallad, A.; Robert, C.; Haanen, J.; Blank, C.; Wesseling, J.; Willems, S. M.; Zecchin, D.; Hobor, S.; Bajpe, P. K.; Lieftink, C.; Mateus, C.; Vagner, S.; Grernrum, W.; Hofland, I.; Schlicker, A.; Wessels, L. F. A.; Beijersbergen, R. L.; Bardelli, A.; Di Nicolantonio, F.; Eggermont, A. M. M.; Bernards, R. Reversible and adaptive resistance to $\mathrm{BRAF}(\mathrm{V} 600 \mathrm{E})$ inhibition in melanoma. Nature 2014, 508 (7494), 118-122.

(21) Dankort, D.; Curley, D. P.; Cartlidge, R. A.; Nelson, B.; Karnezis, A. N.; Damsky, W. E.; You, M. J.; DePinho, R. A.; McMahon, M.; Bosenberg, M. Braf(V600E) cooperates with Pten loss to induce metastatic melanoma. Nat. Genet. 2009, 41 (5), 544-552.

(22) Cheng, W. C.; Tsui, Y. C.; Ragusa, S.; Koelzer, V. H.; Mina, M.; Franco, F.; Laubli, H.; Tschumi, B.; Speiser, D.; Romero, P.; Zippelius, A.; Petrova, T. V.; Mertz, K.; Ciriello, G.; Ho, P. C. Uncoupling protein 2 reprograms the tumor microenvironment to support the anti-tumor immune cycle. Nat. Immunol. 2019, 20 (4), 206-217.

(23) Miller, A. J.; Mihm, M. C., Jr. Melanoma. N. Engl. J. Med. 2006, $355(1), 51-65$.

(24) Zbytek, B.; Carlson, J. A.; Granese, J.; Ross, J.; Mihm, M.; Slominski, A. Current concepts of metastasis in melanoma. Expert Rev. Dermatol. 2008, 3 (5), 569-585.

(25) Yang, Y.; Lin, Y.; Chen, Z. X.; Gong, T. Q.; Yang, P. Y.; Girault, H.; Liu, B. H.; Qiao, L. Bacterial Whole Cell Typing by Mass Spectra Pattern Matching with Bootstrapping Assessment. Anal. Chem. 2017, 89 (22), 12556-12561.

(26) Frank, E.; Hall, M.; Holmes, G.; Kirkby, R.; Pfahringer, B.; Witten, I. H.; Trigg, L. Weka-A Machine Learning Workbench for Data Mining. Data Mining and Knowledge Discovery Handbook; Springer, 2009; pp 1269-1277.
(27) Tsao, H.; Olazagasti, J. M.; Cordoro, K. M.; Brewer, J. D.; Taylor, S. C.; Bordeaux, J. S.; Chren, M. M.; Sober, A. J.; Tegeler, C.; Bhushan, R.; Begolka, W. S. Early detection of melanoma: Reviewing the ABCDEs. J. Am. Acad. Dermatol. 2015, 72 (4), 717-723.

(28) Tran, J. C.; Zamdborg, L.; Ahlf, D. R.; Lee, J. E.; Catherman, A. D.; Durbin, K. R.; Tipton, J. D.; Vellaichamy, A.; Kellie, J. F.; Li, M. X.; Wu, C.; Sweet, S. M. M.; Early, B. P.; Siuti, N.; LeDuc, R. D.; Compton, P. D.; Thomas, P. M.; Kelleher, N. L. Mapping intact protein isoforms in discovery mode using top-down proteomics. Nature 2011, 480 (7376), 254-258.

(29) Makowiecka, A.; Malek, N.; Mazurkiewicz, E.; Mrówczyńska, E.; Nowak, D.; Mazur, A. J. Thymosin $\beta 4$ regulates focal adhesion formation in human melanoma cells and affects their migration and invasion. Front. Cell Dev. Biol. 2019, 7, 304.

(30) Caselitz, J.; Janner, M.; Breitbart, E.; Weber, K.; Osborn, M. Malignant Melanomas Contain Only the Vimentin Type of Intermediate Filaments. Virchows Arch. A: Pathol. Anat. Histol. 1983, 400 (1), 43-51.

(31) Jang, H. S.; Shah, N. M.; Du, A. Y.; Dailey, Z. Z.; Pehrsson, E. C.; Godoy, P. M.; Zhang, D.; Li, D. F.; Xing, X. Y.; Kim, S.; O’Donnell, D.; Gordon, J. I.; Wang, T. Transposable elements drive widespread expression of oncogenes in human cancers. Nat. Genet. 2019, 51 (4), 611-617.

(32) Dong, X. Q.; Han, Y. S.; Sun, Z.; Xu, J. L. Actin Gamma 1, a new skin cancer pathogenic gene, identified by the biological featurebased classification. J. Cell. Biochem. 2018, 119 (2), 1406-1419.

(33) Sgarbi, G.; Barbato, S.; Costanzini, A.; Solaini, G.; Baracca, A. The role of the ATPase inhibitor factor 1 (IF1) in cancer cells adaptation to hypoxia and anoxia. Biochim. Biophys. Acta, Bioenerg. 2018, 1859 (2), 99-109.

(34) Bresnick, A. R.; Weber, D. J.; Zimmer, D. B. S100 proteins in cancer. Nat. Rev. Cancer 2015, 15 (2), 96-109.

(35) Calderwood, S. K.; Gong, J. L. Heat Shock Proteins Promote Cancer: It's a Protection Racket. Trends Biochem. Sci. 2016, 41 (4), 311-323.

(36) Petrova, V.; Annicchiarico-Petruzzelli, M.; Melino, G.; Amelio, I. The hypoxic tumour microenvironment. Oncogenesis 2018, 7, 10.

(37) Lundgren, D. H.; Hwang, S. I.; Wu, L. F.; Han, D. K. Role of spectral counting in quantitative proteomics. Expert Rev. Proteomics 2010, 7 (1), 39-53.

(38) Weinstein, D.; Leininger, J.; Hamby, C.; Safai, B. Diagnostic and prognostic biomarkers in melanoma. J. Clin. Aesthet. Dermatol. 2014, 7 (6), 13-24.

(39) Troyanova-Wood, M.; Meng, Z. K.; Yakovlev, V. V. Differentiating melanoma and healthy tissues based on elasticityspecific Brillouin microspectroscopy. Biomed. Opt. Express 2019, 10 (4), 1774-1781.

(40) Sarna, M.; Zadlo, A.; Czuba-Pelech, B.; Urbanska, K. Nanomechanical Phenotype of Melanoma Cells Depends Solely on the Amount of Endogenous Pigment in the Cells. Int. J. Mol. Sci. 2018, 19 (2), 607.

(41) Alderton, G. K. DIAGNOSIS Fishing for exosomes. Nat. Rev. Cancer 2015, 15 (8), 453-453.

(42) Zhu, Y. D.; Pick, H.; Gasilova, N.; Li, X. Y.; Lin, T. E.; Laeubli, H. P.; Zippelius, A.; Ho, P. C.; Girault, H. H. MALDI Detection of Exosomes: A Potential Tool for Cancer Studies. Chem-Us 2019, 5 (5), $1318-1336$.

(43) Jain, S.; Gautam, V.; Naseem, S. Acute-phase proteins: As diagnostic tool. J. Pharm. BioAllied Sci. 2011, 3 (1), 118.

(44) Dowling, P.; Clarke, C.; Hennessy, K.; Torralbo-Lopez, B.; Ballot, J.; Crown, J.; Kiernan, I.; O'Byrne, K. J.; Kennedy, M. J.; Lynch, V.; Clynes, M. Analysis of acute-phase proteins, AHSG, C3, CLI, HP and SAA, reveals distinctive expression patterns associated with breast, colorectal and lung cancer. Int. J. Cancer 2012, 131 (4), 911-923.

(45) Pirok, B. W.; Molenaar, S. R.; Roca, L. S.; Schoenmakers, P. J. Peak-tracking algorithm for use in automated interpretive methoddevelopment tools in liquid chromatography. Anal. Chem. 2018, 90 (23), 14011-14019. 
(46) Hardesty, W. M.; Kelley, M. C.; Mi, D. M.; Low, R. L.; Caprioli, R. M. Protein signatures for survival and recurrence in metastatic melanoma. J. Proteomics 2011, 74 (7), 1002-1014.

(47) Caputo, E.; Maiorana, L.; Vasta, V.; Pezzino, F. M.; Sunkara, S.; Wynne, K.; Elia, G.; Marincola, F. M.; McCubrey, J. A.; Libra, M.; Travali, S.; Kane, M. Characterization of human melanoma cell lines and melanocytes by proteome analysis. Cell Cycle 2011, 10 (17), 2924-2936.

(48) Inkeles, M. S.; Scumpia, P. O.; Swindell, W. R.; Lopez, D.; Teles, R. M.B.; Graeber, T. G.; Meller, S.; Homey, B.; Elder, J. T.; Gilliet, M.; Modlin, R. L.; Pellegrini, M. Comparison of molecular signatures from multiple skin diseases identifies mechanisms of immunopathogenesis. J. Invest. Dermatol. 2015, 135 (1), 151-159.

(49) Shih, B. B.; Nirmal, A. J.; Headon, D. J.; Akbar, A. N.; Mabbott, N. A.; Freeman, T. C. Derivation of marker gene signatures from human skin and their use in the interpretation of the transcriptional changes associated with dermatological disorders. Journal of Pathology 2017, 241 (5), 600-613.

(50) Brown, R. S.; Lennon, J. J. Mass Resolution Improvement by Incorporation of Pulsed Ion Extraction in a Matrix-Assisted LaserDesorption Ionization Linear Time-of-Flight Mass-Spectrometer. Anal. Chem. 1995, 67 (13), 1998-2003.

(51) López-Fernández, H.; Santos, H. M.; Capelo, J. L.; FdezRiverola, F.; Glez-Peña, D.; Reboiro-Jato, M. Mass-Up: an all-in-one open software application for MALDI-TOF mass spectrometry knowledge discovery. BMC Bioinformatics 2015, 16, 318. 